\title{
2PI effective action for the SYK model and tensor field theories
}

\author{
Dario Benedetti $^{a}$ and Razvan Gurau ${ }^{b, c}$ \\ ${ }^{a}$ Laboratoire de Physique Théorique (UMR 8627), CNRS, Université Paris-Sud, \\ Université Paris-Saclay, 91405 Orsay, France \\ ${ }^{b}$ CPHT, Ecole Polytechnique, CNRS, Université Paris-Saclay, \\ Route de Saclay, 91128 Palaiseau, France \\ ${ }^{c}$ Perimeter Institute for Theoretical Physics, \\ 31 Caroline St. N, N2L 2Y5, Waterloo, ON, Canada \\ E-mail: dario.benedetti@th.u-psud.fr, rgurau@cpht.polytechnique.fr
}

ABSTRACT: We discuss the two-particle irreducible (2PI) effective action for the SYK model and for tensor field theories. For the SYK model the 2PI effective action reproduces the bilocal reformulation of the model without using replicas.

In general tensor field theories the 2PI formalism is the only way to obtain a bilocal reformulation of the theory, and as such is a precious instrument for the identification of soft modes and for possible holographic interpretations. We compute the 2PI action for several models, and push it up to fourth order in the $1 / N$ expansion for the model proposed by Witten in [1], uncovering a one-loop structure in terms of an auxiliary bilocal action.

Keywords: 1/N Expansion, Field Theories in Lower Dimensions

ARXIV EPRINT: 1802.05500 


\section{Contents}

1 Introduction 1

2 2PI effective action for vector models 3

2.1 Large- $N$ expansion 8

2.2 Large- $N$ expansion as the loop expansion of an auxiliary theory 12

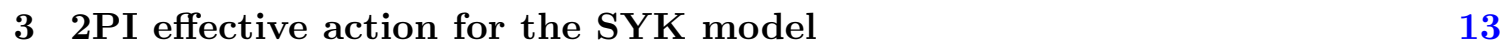

$\begin{array}{lll}3.1 & \text { Next-to-leading order action } & 16\end{array}$

$\begin{array}{lll}4 & \text { 2PI effective action for tensor field theories } & 18\end{array}$

$\begin{array}{ll}4.1 \text { The bosonic CTKT model in } d=0 & 19\end{array}$

4.2 The fermionic CTKT model in $d=1 \quad 22$

$\begin{array}{ll}\text { 4.3 The fermionic GW model in } d=1 & 25\end{array}$

5 Summary and outlook $\quad 31$

$\begin{array}{ll}\text { A Orthogonal decomposition of the fluctuations } & 31\end{array}$

\section{Introduction}

The Sachdev-Ye-Kitaev (SYK) model [2,3], which is a one-dimensional model of $N$ Majorana fermions with quenched random couplings, has recently been intensely studied as a testbed for the AdS/CFT duality [4-7]. Arguably, one of the most useful features of the model is that at the first few orders in $1 / N$ it can be reformulated as a bilocal field theory $[3,8,9]$. This allows to: derive the large- $N$ Schwinger-Dyson equations as equations of motion [3]; neatly identify the light mode associated to the non-conformal perturbation in the strong coupling limit and derive its effective dynamics (controlled by the so-called Schwarzian action [5, 7, 9], which can then be matched to a possible gravitational dual [10-12]); efficiently build a perturbative expansion for $n$-point functions of bilinear operators [7, 13-15]; possibly provide a holographic interpretation along the lines of $[16,17]$. However, the bilocal theory lives in a replica space and it is difficult (and not done in the literature) to study the fluctuations that break replica symmetry. Consequently, it only works at the first few orders in $1 / N$ at which the quenched and annealed versions of the model coincide [7, 18].

In 2016 Witten [1] proposed a tensor model $[19,20]$ with a similar large- $N$ limit as the SYK model, but without quenched disorder. The fact that this model is a genuine quantum system and has a symmetry which can be gauged (thus allowing to restrict the operators in the theory to be singlets) makes it in principle more appealing from the point 
of view of holography. For these reasons, this model and other similar ones have been intensely studied at large $N[21-31]$, and at finite $N$ [32-35]. The tensor large- $N$ limit has also been used to derive a new large- $D$ expansion for multi-matrix models [36-39].

While they coincide at leading order, the SYK model and its tensor analogues are quite different at subleading orders [40]. Moreover, tensor field theories ${ }^{1}$ have many more covariant and invariant (or singlet) operators [25, 44-46]. This has rather drastic consequences: one-dimensional tensor models display a large number of light modes in the infrared $[25,27]$ which are absent in the SYK model. In order to study these modes, and possibly to better understand the holographic dual of such theories, it would be useful to have a bilocal reformulation of the theory. In fact, while the construction of the bilocal action in the SYK model is a standard procedure for disordered systems such as spin glasses [47, 48], it is not tied to disorder and it can also be understood in a more general context as a special case of the collective field method [49]. However, until now, no collective field formulation has been found for tensor models (except in the few cases in which an intermediate field representation is possible, but which do not have SYK-type behavior [29]): in [27] a bilocal action is postulated but not derived, while in [25] the existence of the new light modes is inferred from other arguments; in [50] a bilocal action is proposed for the Gurau-Witten model [1, 19], but it leads to wrong Schwinger-Dyson equations. Actually, one can expect that no simple and exact reformulation of tensor models is possible in terms of few collective variables because the collective field method of [49] is based on the idea that one could rewrite a theory with a certain symmetry directly in terms of its invariants; but while vector models have only one possible invariant (and its derivatives), and matrix models can be reduced to eigenvalues, which are much less than the original number of variables, tensor models have a much larger number of invariants and no useful reduction to eigenvalues is known. Furthermore, for vector models the collective field reformulation reduces the large- $N$ expansion to a simple saddle-point (or loop) expansion, which we do not expect to be the case for tensor models.

In this paper we propose to use the two-particle irreducible (2PI) effective action formalism [51] (see [52] for a modern review) for tensor models and show that it provides a useful version of the bilocal reformulation. The $2 \mathrm{PI}$ formalism has been applied to a variety of problems (see for example [53-56] and references therein) and it has been shown to be well suited for a $1 / N$ expansion in the case of the $O(N)$ model $[57,58]$. Nevertheless, it is not one of the most popular formalisms around, and therefore we will review it in a self-contained way in section 2, together with its large- $N$ expansion for the $O(N)$ model in section 2.1. The connection to the collective field formalism is in this case straightforward, as we will explain in section 2.2 .

In order to elucidate the usefulness of the 2PI effective action it is worthwhile to apply it to the SYK model first, as we will do in section 3. It turns out that the 2 PI reformulation reproduces exactly all the results of $[5,7,9]$, up to the same order in $1 / N$ without using

\footnotetext{
${ }^{1}$ In this paper, tensor field theories are local field theories whose fields transform as a tensor under a global or local symmetry group. They should not be confused with the theories that go under the same name [41, 42] (also sometimes referred to as tensorial group field theories [43]) but which are field theories with a non-local interaction following a tensorial pattern of points identification.
} 
the replica method, i.e. without using the trick in eq. (3.19). This being said, the 2PI reformulation in the SYK model has its own drawbacks:

- it requires to know explicitly the graphs contributing to each order in $1 / N$. While this is exogenous to the formalism, hence not very aesthetically pleasing, the graph analysis has already been done and we are able to use this information to write the 2PI action up to the same order as the usual replica based bilocal action.

- it also fails at higher enough orders in $1 / N$. This has nothing to do with replicas, although it happens at the same order at which the replica diagonal ansatz breaks down: it has to do with the lack of commutation between going on shell and taking quenched averages (this will be explained in section 3).

The main lesson to be drawn from the 2PI reformulation of the SYK model is that the leading and next-to-leading orders in the $1 / N$ expansion of the model are exactly the leading order and first loop correction in a loop expansion of the bilocal theory of $[5,7,9]$. This structure does not survive at higher orders: the $1 / N$ expansion is a loop expansion in the annealed version of the model, but not in the quenched one.

The main point of our paper is however that the 2PI formalism becomes much more useful in the tensor case, where the issues with the quenched average are absent, and where we do not yet have an alternative collective field reformulation. We will apply it to the Gurau-Witten [1, 19] and the Carrozza-Tanasa-Klebanov-Tarnopolsky [22, 59] models in section 4. Among other things we will in this way put on a firmer ground the result of [27] by showing that the bilocal action that they postulated is in fact the leading-order 2PI effective action. Furthermore, in the Gurau-Witten model we will be able to expand the action up to fourth order in the $1 / N$ expansion, highlighting a similar structure among the three subleading terms: they all have the form of a logarithm of a determinant, hence they can be interpreted as Gaussian integrals over bilocal fields. Surprisingly, we will see that all such terms can be interpreted as the one-loop correction of an auxiliary bilocal effective action.

\section{$2 \quad 2$ PI effective action for vector models}

Let us review the definition and properties of the 2PI effective action [51]. We consider a theory of real bosonic scalar fields $\varphi_{\mathbf{a}}$, where the index $\mathbf{a}$ denotes both a space time point and flavor indices ${ }^{2}$ with classical action $\mathbf{S}[\varphi]$. We denote functionals by capital bold letters and, in order to simplify notation, we will sometimes omit the arguments of functionals. Sums, products, Kronecker deltas and traces include both flavor indices and space time points, and repeated indices are summed. We define:

$$
\mathbf{W}[j, k]=\ln \int[d \varphi] \exp \left\{-\mathbf{S}[\varphi]+j_{\mathbf{a}} \varphi_{\mathbf{a}}+\frac{1}{2} \varphi_{\mathbf{a}} k_{\mathbf{a b}} \varphi_{\mathbf{b}}\right\},
$$

\footnotetext{
${ }^{2}$ For example, vector indices $a$ when the fields form a vector representation of some group. For specific models later in the paper we will make the distinction between space time points and other indices explicit, writing for example $\varphi_{\mathbf{a}}=\varphi_{a}(x)$.
} 
which is the generating functional of connected moments of a theory with shifted inverse covariance $\frac{\delta^{2} \mathbf{S}}{\delta \varphi_{\mathbf{a}} \delta \varphi_{\mathbf{b}}}[0]-k_{\mathbf{a b}}$. Observe that $\mathbf{W}[j, k]$ depends only on the symmetric part of $k_{\mathrm{ab}}$ which then is assumed to be symmetric in its indices. Therefore:

$$
\frac{\delta k_{\mathrm{ab}}}{\delta k_{\mathrm{mn}}}=\frac{1}{2} \mathcal{S}_{\mathrm{ab} ; \mathbf{m n}}
$$

where we have introduced the projector on symmetric matrices: ${ }^{3}$

$$
\mathcal{S}_{\mathbf{a b} ; \mathbf{m n}}=\frac{1}{2}\left(\delta_{\mathbf{a m}} \delta_{\mathbf{b n}}+\delta_{\mathbf{a n}} \delta_{\mathbf{b m}}\right) .
$$

To simplify notation we will denote sometimes the functional derivatives as indices:

$$
\mathbf{W}_{j_{\mathbf{a}}}[j, k] \equiv \frac{\delta \mathbf{W}}{\delta j_{\mathbf{a}}}[j, k], \quad \mathbf{W}_{k_{\mathbf{a b}}}[j, k] \equiv \frac{\delta \mathbf{W}}{\delta k_{\mathbf{a b}}}[j, k]
$$

Using two independent sources in (2.1) has the advantage that one obtains several expressions for the correlations of the theory in terms of derivatives of the generating functions, which gives a certain redundancy in the description of the theory in this language: derivatives with respect to $k_{\mathbf{a b}}$ are related to repeated derivatives with respect to $j_{\mathbf{a}}$, the examples of one and two $k$-derivatives being explicitly worked out below. Such relations could be used as consistency checks for truncations of the generating functional which are performed in some approximation schemes where there is no small parameter, but such checks will not be needed for more systematic approximation schemes, such as the $1 / N$ expansion that we will discuss below. In the following, we will use as much as possible only derivatives with respect to the bilocal source to express the interesting correlations, hence we will often put the local source on shell very early on. Alternatively one could start from the beginning by introducing only the bilocal source $k$. This has the drawback that not all the correlations of the theory can be obtained from the generating functional. However, all the ones which interest us in this paper can.

We denote $\mathbf{\Phi}$ and $\mathbf{G}$ the connected 1-point and 2-point functions of the theory with sources $j$ and $k$ :

$$
\begin{aligned}
\boldsymbol{\Phi}_{\mathbf{a}}[j, k] & =\mathbf{W}_{j_{\mathbf{a}}}[j, k], \\
\mathbf{G}_{\mathbf{a b}}[j, k]=\mathbf{W}_{j_{\mathbf{a}} j_{\mathbf{b}}}[j, k] & =2 \mathbf{W}_{k_{\mathbf{a b}}}[j, k]-\mathbf{W}_{j_{\mathbf{a}}}[j, k] \mathbf{W}_{j_{\mathbf{b}}}[j, k] .
\end{aligned}
$$

We are generally interested in the connected 1-point and 2-point functions of the theory without sources, for which we introduce the following notation:

$$
\mathbf{\Phi}_{\mathbf{a}}[0,0]=\left\langle\varphi_{\mathbf{a}}\right\rangle_{\mathrm{conn}} \equiv \underline{\phi}_{\mathbf{a}}, \quad \mathbf{G}_{\mathbf{a b}}[0,0]=\left\langle\varphi_{\mathbf{a}} \varphi_{\mathbf{b}}\right\rangle_{\mathrm{conn}} \equiv \underline{G}_{\mathbf{a b}}
$$

Notice that $\mathbf{G}_{\mathbf{a b}}$ (hence in particular $\underline{G}_{\mathbf{a b}}$ ) is symmetric in its indices.

\footnotetext{
${ }^{3}$ In the case of Grassmann fields (for which we will typically use the letters $\psi$ and $\Psi$ instead of $\varphi$ and $\phi) k_{\mathbf{a b}}$ is antisymmetric, hence the derivative evaluates to the antisymmetric projector: $\mathcal{A}_{\mathbf{a b} ; \mathbf{m n}}=$ $\frac{1}{2}\left(\delta_{\mathrm{am}} \delta_{\mathrm{bn}}-\delta_{\mathrm{an}} \delta_{\mathrm{bm}}\right)$.
} 
For a free theory with covariance $C$ we obtain:

$$
\mathbf{W}^{C}[j, k]=-\frac{1}{2} \operatorname{Tr}\left[\ln \left(C^{-1}-k\right)\right]+\frac{1}{2} j_{\mathbf{a}}\left(\frac{1}{C^{-1}-k}\right)_{\mathbf{a b}} j_{\mathbf{b}} .
$$

and as a consequence:

$$
\mathbf{\Phi}_{\mathbf{a}}^{C}[j, k]=\left(\frac{1}{C^{-1}-k}\right)_{\mathbf{a b}} j_{\mathbf{b}}, \quad \mathbf{G}_{\mathbf{a b}}^{C}[j, k]=\left(\frac{1}{C^{-1}-k}\right)_{\mathbf{b a}} .
$$

Let $\left\{\mathbf{J}_{\mathbf{a}}[\phi, G], \mathbf{K}_{\mathbf{a b}}[\phi, G]\right\}$ be the inverse of $\left\{\boldsymbol{\Phi}_{\mathbf{a}}[j, k], \mathbf{G}_{\mathbf{a b}}[j, k]\right\}$. For a free theory they are:

$$
\mathbf{J}_{\mathbf{a}}^{C}[\phi, G]=\left(G^{-1}\right)_{\mathbf{a b}} \phi_{\mathbf{b}} \quad \mathbf{K}_{\mathbf{a b}}^{C}[\phi, G]=\left(C^{-1}\right)_{\mathbf{a b}}-\left(G^{-1}\right)_{\mathbf{a b}} .
$$

The 1- and 2-point functions $\underline{\phi}$ and $\underline{G}$ are then determined implicitly by the equations:

$$
\mathbf{J}_{\mathbf{a}}[\underline{\phi}, \underline{G}]=0, \quad \mathbf{K}_{\mathbf{a b}}[\underline{\phi}, \underline{G}]=0,
$$

and for the free theory we get $\underline{\phi}^{C}=0, \underline{G}^{C}=C$.

The second derivative of $\overline{\mathbf{W}}$ with respect to $k$ is:

$$
\mathbf{W}_{k_{\mathrm{ab}} k_{\mathrm{cd}}}=\frac{1}{2} \mathbf{G}_{\mathbf{a b} ; k_{\mathrm{cd}}}+\frac{1}{2} \boldsymbol{\Phi}_{\mathbf{a} ; k_{\mathrm{cd}}} \boldsymbol{\Phi}_{\mathbf{b}}+\frac{1}{2} \boldsymbol{\Phi}_{\mathbf{a}} \boldsymbol{\Phi}_{\mathbf{b} ; k_{\mathrm{cd}}} .
$$

Assuming that we are in a symmetric phase in which the 1-point function is zero, $\underline{\phi}=0$, we obtain:

$$
\sum_{\mathbf{c d}} \mathbf{W}_{k_{\mathbf{a b}} k_{\mathbf{c d}}}[j=0, k=0] \mathbf{K}_{\mathbf{c d} ; G_{\mathbf{m n}}}[\underline{\phi}=0, \underline{G}]=\frac{1}{2} \frac{\delta G_{\mathbf{a b}}}{\delta G_{\mathbf{m n}}}=\frac{1}{2} S_{\mathbf{a b} ; \mathbf{m n}} .
$$

The connected 4-point function is the fourth derivative $\mathbf{W}_{j_{\mathbf{a}} j_{\mathbf{b}} j_{\mathbf{c}} j_{\mathbf{d}}}[0,0]$. It can be re expressed using derivatives with respect to $k$, as several relations exist between derivatives of $\mathbf{W}[j, k]$ with respect to $j$ and $k$. The simplest one which is obtained by noticing that deriving the partition function $\exp \{\mathbf{W}[j, k]\}$ once with respect to $k$ we obtain (one half times) the same result as deriving twice with respect to $j$ :

$$
\left(\mathbf{W}_{j_{\mathrm{a}} j_{\mathbf{b}}}+\mathbf{W}_{j_{\mathbf{a}}} \mathbf{W}_{j_{\mathbf{b}}}-2 \mathbf{W}_{k_{\mathrm{ab}}}\right) e^{\mathbf{W}}=0
$$

leading to eq. (2.6). Deriving this equality either one more time with respect to $k$ or two more times with respect to $j$, and combining the results we obtain a long relation, which simplifies considerably in a symmetric phase $\underline{\phi}=0$ :

$$
\mathbf{W}_{k_{\mathbf{a b}} k_{\mathbf{c d}}}[0,0]=\frac{1}{4}\left(\mathbf{W}_{j_{\mathbf{a}} j_{\mathbf{b}} j_{\mathbf{c}} j_{\mathbf{d}}}+\mathbf{W}_{j_{\mathbf{a}} j_{\mathbf{c}}} \mathbf{W}_{j_{\mathbf{b}} j_{\mathbf{d}}}+\mathbf{W}_{j_{\mathbf{a}} j_{\mathbf{d}}} \mathbf{W}_{j_{\mathbf{b}} j_{\mathbf{c}}}\right)_{j, k=0} \equiv \frac{1}{4} \mathcal{F}(\mathbf{a}, \mathbf{b}) ;(\mathbf{c}, \mathbf{d})
$$

We have thus obtained an explicit relation between the derivatives of $\mathbf{W}$ (on shell) with respect to the $k$ and $j$ sources, as advertised before. The function $\mathcal{F}_{(\mathbf{a}, \mathbf{b}) ;(\mathbf{c}, \mathbf{d})}$ is the full 4point function minus the contribution of the disconnected channel $(\mathbf{a}, \mathbf{b})(\mathbf{c}, \mathbf{d})$. For example, in the free theory we obtain from (2.6) and (2.9):

$$
\mathbf{W}_{k_{\mathbf{a b}} k_{\mathbf{c d}}}^{C}[0,0]=\frac{1}{2} C_{\mathbf{b m}} C_{\mathbf{m a}} S_{\mathbf{m n} ; \mathbf{c d}}=\frac{1}{4}\left(C_{\mathbf{b c}} C_{\mathbf{d a}}+C_{\mathbf{b d}} C_{\mathbf{c a}}\right) .
$$


We define the 2PI effective action ${ }^{4}$ of the theory as the double Legendre transform of $\mathbf{W}[j, k]$ :

$$
\boldsymbol{\Gamma}[\phi, G]=-\mathbf{W}[\mathbf{J}, \mathbf{K}]+\mathbf{J}_{\mathbf{a}} \phi_{\mathbf{a}}+\frac{1}{2} \phi_{\mathbf{a}} \mathbf{K}_{\mathbf{a b}} \phi_{\mathbf{b}}+\frac{1}{2} \operatorname{Tr}[G \mathbf{K}] .
$$

Deriving (2.16) with respect to $\phi$ and $G$, we obtain the two identities:

$$
\boldsymbol{\Gamma}_{\phi_{\mathbf{a}}}[\phi, G]=\mathbf{J}_{\mathbf{a}}[\phi, G]+\mathbf{K}_{\mathbf{a b}}[\phi, G] \phi_{\mathbf{b}}, \quad \boldsymbol{\Gamma}_{G_{\mathbf{a b}}}[\phi, G]=\frac{1}{2} \mathbf{K}_{\mathbf{b a}}[\phi, G] .
$$

Furthermore, $\boldsymbol{\Gamma}_{G G}[\phi, G]=\frac{1}{2} \mathbf{K}_{G}[\phi, G]$ which, combined with eq. (2.12) and (2.14), yields for a theory in the symmetric phase:

$$
\mathcal{F}_{(\mathbf{a}, \mathbf{b}) ;(\mathbf{c}, \mathbf{d})} \boldsymbol{\Gamma}_{G_{\mathbf{c d}} G_{\mathbf{m n}}}[0, \underline{G}]=\mathcal{S}_{\mathbf{a b} ; \mathbf{m n}} .
$$

As usual we get back to $\mathbf{W}[j, k]$ by means of a new Legendre transform:

$$
\mathbf{W}[j, k]=-\boldsymbol{\Gamma}[\mathbf{\Phi}, \mathbf{G}]+j_{\mathbf{a}} \boldsymbol{\Phi}_{\mathbf{a}}+\frac{1}{2} \boldsymbol{\Phi}_{\mathbf{a}} k_{\mathbf{a b}} \boldsymbol{\Phi}_{\mathbf{b}}+\frac{1}{2} \operatorname{Tr}[\mathbf{G} k],
$$

where the functionals $\mathbf{\Phi}[j, k], \mathbf{G}[j, k]$ are determined by solving:

$$
\boldsymbol{\Gamma}_{\phi}[\mathbf{\Phi}, \mathbf{G}]=j+k \mathbf{\Phi}, \quad \boldsymbol{\Gamma}_{G}[\mathbf{\Phi}, \mathbf{G}]=\frac{1}{2} k .
$$

The 2PI effective action has a number of interesting features [51, 52]:

1. The solution of the equations of motion $\boldsymbol{\Gamma}_{\phi}=0, \boldsymbol{\Gamma}_{G}=0$ is $\underline{\phi}, \underline{G}$, which are the connected 1- and 2-point functions of the theory.

2. It can be evaluated in a loop expansion. Substituting $\mathbf{J}[\phi, G]$ and $\mathbf{K}[\phi, G]$ for $j$ and $k$ into (2.1), and using eq. (2.16), we obtain:

$$
e^{-\Gamma[\phi, G]+\mathbf{J} \phi+\frac{1}{2} \phi \mathbf{K} \phi+\frac{1}{2} \operatorname{Tr}[G \mathbf{K}]}=\int[d \varphi] e^{-\mathbf{S}[\varphi]+\mathbf{J} \varphi+\frac{1}{2} \varphi \mathbf{K} \varphi} .
$$

We translate $\varphi \rightarrow \phi+\varphi$ and expand

$$
\mathbf{S}[\phi+\varphi]=\mathbf{S}[\phi]+\mathbf{S}_{\phi}[\phi] \varphi+\frac{1}{2} \varphi \mathbf{S}_{\phi \phi}[\phi] \varphi+\mathbf{S}_{\mathrm{int}}[\phi, \varphi],
$$

where the interacting part of the action $\mathbf{S}_{\text {int }}[\phi, \varphi]$ contains all higher powers of $\varphi$. We obtain:

$$
e^{-\boldsymbol{\Gamma}[\phi, G]+\frac{1}{2} \operatorname{Tr}[G \mathbf{K}]}=\int[d \varphi] e^{-\mathbf{S}[\phi]+\left(\mathbf{J}+\mathbf{K} \phi-\mathbf{S}_{\phi}[\phi]\right) \varphi-\frac{1}{2} \varphi\left(\mathbf{S}_{\phi \phi}[\phi]-\mathbf{K}\right) \varphi-\mathbf{S}_{\mathrm{int}}[\phi, \varphi]},
$$

or, using (2.17):

$$
e^{-\boldsymbol{\Gamma}[\phi, G]+\operatorname{Tr}\left[G \boldsymbol{\Gamma}_{G}[\phi, G]\right]}=\int[d \varphi] e^{-\mathbf{S}[\phi]+\left(\boldsymbol{\Gamma}_{\phi}[\phi, G]-\mathbf{S}_{\phi}[\phi]\right) \varphi-\varphi\left(\frac{1}{2} \mathbf{S}_{\phi \phi}[\phi]-\boldsymbol{\Gamma}_{G}[\phi, G]\right) \varphi-\mathbf{S}_{\mathrm{int}}[\phi, \varphi]} .
$$

\footnotetext{
${ }^{4}$ The reason for this name will become clear below.
} 
So far eq. (2.22) is exact. In order to evaluate it at one loop, we observe that at the classical level $\boldsymbol{\Gamma}[\phi, G] \approx \mathbf{S}[\phi]$, hence $\mathbf{J}+\mathbf{K} \phi-\mathbf{S}_{\phi}[\phi]=\boldsymbol{\Gamma}_{\phi}-\mathbf{S}_{\phi}$ is already at one loop and the linear term in the Gaussian integral can be neglected as it yields a two loop effect upon integration over $\varphi$. Thus at one loop we have:

$$
\boldsymbol{\Gamma}_{1}[\phi, G]=\mathbf{S}[\phi]+\frac{1}{2} \operatorname{Tr}\left[G \mathbf{K}_{1}\right]+\frac{1}{2} \operatorname{Tr} \ln \left(\mathbf{S}_{\phi \phi}[\phi]-\mathbf{K}_{1}\right),
$$

where $\mathbf{K}_{1}$ is the functional $\mathbf{K}[\phi, G]=2 \boldsymbol{\Gamma}_{G}[\phi, G]$ evaluated at one loop. On the other hand, at one loop:

$$
\frac{\delta \boldsymbol{\Gamma}_{1}[\phi, G]}{\delta G}-\frac{1}{2} \mathbf{K}_{1}=\frac{1}{2}\left(G-\frac{1}{\mathbf{S}_{\phi \phi}[\phi]-\mathbf{K}_{1}}\right) \frac{\delta \mathbf{K}_{1}}{\delta G}=0,
$$

which in turn fixes $\mathbf{K}_{1}=\mathbf{S}_{\phi \phi}[\phi]-G^{-1}$. Substituting this in eq. (2.24) and discarding a constant term we obtain: ${ }^{5}$

$$
\boldsymbol{\Gamma}[\phi, G]=\mathbf{S}[\phi]+\frac{1}{2} \operatorname{Tr}\left[\ln G^{-1}\right]+\frac{1}{2} \operatorname{Tr}\left[G_{0}^{-1} G\right]+\boldsymbol{\Gamma}_{2}[\phi, G],
$$

where $G_{0}=\left(\mathbf{S}_{\phi \phi}[\phi]\right)^{-1}$ is the free covariance of the theory around the field configuration $\phi$ and $\boldsymbol{\Gamma}_{2}[\phi, G]$ starts at two loops.

In the free theory of covariance $C$, the one loop result is exact, and therefore we have:

$$
\boldsymbol{\Gamma}^{C}[\phi, G]=\frac{1}{2} \phi_{\mathbf{a}} C_{\mathbf{a b}}^{-1} \phi_{\mathbf{b}}+\frac{1}{2} \operatorname{Tr}\left[\ln G^{-1}\right]+\frac{1}{2} \operatorname{Tr}\left[C^{-1} G\right],
$$

and it can be easily verified that (2.18) holds.

3. The equations of motion of (2.26) with respect to $G$ write:

$$
G^{-1}=G_{0}^{-1}+2 \frac{\delta \boldsymbol{\Gamma}_{2}}{\delta G}
$$

As $G_{0}$ is the free covariance of the theory and $G$ is the connected two point function, it follows form the standard Schwinger-Dyson equation $G^{-1}=G_{0}^{-1}-\Sigma$ that $-2 \frac{\delta \boldsymbol{\Gamma}_{2}}{\delta G}$ must be identified with the self energy $\Sigma$ of the model, which is the sum of amputated one-particle-irreducible two point graphs.

4. $\boldsymbol{\Gamma}_{2}[\phi, G]$ is given by (minus) the sum of all the two-particle irreducible vacuum graphs (i.e. graphs that do not disconnect when cutting open any two edges) with vertices given by the effective interaction $\mathbf{S}_{\text {int }}[\phi, \varphi]$ and effective propagators $G$. This is slightly non trivial. From eq. $(2.22)$ we see that $\boldsymbol{\Gamma}[\phi, G]$ is the sum of connected graphs with:

- trivalent or higher order vertices given by $-\mathbf{S}_{\text {int }}[\phi, \varphi]$,

- univalent vertices $\varphi\left(\mathbf{J}+\mathbf{K} \phi-\mathbf{S}_{\phi}[\phi]\right)$,

\footnotetext{
${ }^{5}$ Repeating the same constructions for complex or Grassmann fields, it is easy to see that we arrive at a similar expression, but with the functional trace terms multiplied by an extra factor 2 for the complex case and by a minus for the Grassmann case.
} 
- propagators $\left(\mathbf{S}_{\phi \phi}[\phi]-\mathbf{K}\right)^{-1}$,

- a vacuum term $\frac{1}{2} \operatorname{Tr}[G \mathbf{K}]$.

On the other hand, as $-2 \frac{\delta \boldsymbol{\Gamma}_{2}}{\delta G}=\Sigma$ it follows that $\boldsymbol{\Gamma}_{2}$ can be reconstructed by reconnecting the two external vertices of the self energy by a propagator $G$, and since $\Sigma$ is one-particle-irreducible (1PI), $\boldsymbol{\Gamma}_{2}$ is two-particle-irreducible (2PI).

Two questions arise:

- what happened to the univalent vertices? As $\boldsymbol{\Gamma}_{2}$ is $2 \mathrm{PI}$ it is in particular 1PI, hence can not have any univalent vertices. What happens is that the perturbative expansion of $\boldsymbol{\Gamma}[\phi, G]$ in eq. (2.22) for generic $\mathbf{J}$ and $\mathbf{K}$ is built out of connected graphs. Each connected graph has the structure of a tree connecting 1PI vertex kernels. At the self-consistent values of the sources $\mathbf{J}$ and $\mathbf{K}$, obtained from (2.17), the univalent vertices $\varphi(\mathbf{J}+\mathbf{K} \phi)$ act as counter terms and subtract the contribution of all the trees with more that one 1PI vertex kernel.

- the covariance of the theory is $\left(\mathbf{S}_{\phi \phi}[\phi]-\mathbf{K}\right)^{-1}$, so why are the edges of the $2 \mathrm{PI}$ graphs contributing to $\boldsymbol{\Gamma}_{2}$ decorated by $G$ ? The 1PI kernels can still be twoparticle reducible. However, one can resum all the two-point function corrections and replace the propagators by the full two point function of the theory which is $G$. Using the resummed two point function makes the graphs $2 \mathrm{PI}$.

In summary we can write the schematic expression:

$$
e^{-\boldsymbol{\Gamma}[\phi, G]}=e^{-\mathbf{S}[\phi]-\frac{1}{2} \operatorname{Tr}\left[G_{0}^{-1} G\right]} \int_{2 P I}[d \varphi] e^{-\frac{1}{2} \varphi G^{-1} \varphi-\mathbf{S}_{\mathrm{int}}[\phi, \varphi]},
$$

where the subscript 2PI reminds us that in the perturbative expansion of the functional integral we only retain 2PI graphs.

\subsection{Large- $N$ expansion}

While the properties listed above are completely generic, we are now going to review a useful expansion of the 2PI effective action that is applicable to certain models, namely the $1 / N$ expansion. We will use a classical example [51, 52], the $O(N)$ model, in which $N$ is the number of scalar fields: $\varphi_{a}(x)$, with $a=1, \ldots, N$. From now on, we make explicit the vector indices and the space time points. We write $\int_{x}=\int d^{d} x, \int_{x, y}=\int d^{d} x d^{d} y$, and so on and we denote by $\operatorname{Tr}$ a trace both on vector indices and a functional trace, i.e. for a matrix-valued bi-local field $F_{a b}(x, y)$ we have

$$
\operatorname{Tr}[F]=\int_{x, y} \delta(x-y) \operatorname{Tr}\left[F_{a b}(x, y)\right]=\int_{x} F_{a a}(x, x) .
$$

As before, summation is implicit on repeated indices.

In the $O(N)$ model, the $N$ scalars are postulated to transform in the fundamental representation of the $O(N)$ group, i.e.

$$
\varphi_{a}(x) \rightarrow R_{a b} \varphi_{b}(x), \quad R \in O(N),
$$



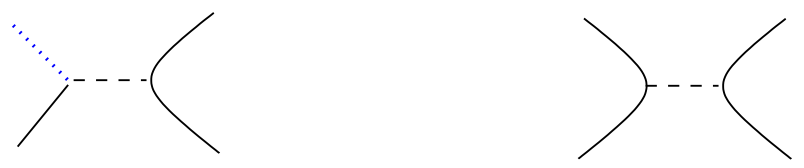

Figure 1. The two vertices from eq. (2.33).

and the action is chosen to be invariant under such transformations. More specifically, restricting to quartic interactions, the action is:

$$
\mathbf{S}[\varphi]=\frac{1}{2} \int_{x, y} \varphi_{a}(x) C^{-1}(x, y) \varphi_{a}(y)+\frac{\lambda}{4 ! N} \int_{x}\left(\varphi_{a}(x) \varphi_{a}(x)\right)^{2},
$$

where $C(x, y)$ is the covariance of the Gaussian functional measure of the free theory. In $d \geq 1, C^{-1}(x, y)$ is usually the kernel of a differential operator, e.g. $C^{-1}=-\partial^{2}+m^{2}$.

All the definitions we introduced above for the 2PI effective action apply directly, with:

$$
\begin{aligned}
G_{0, a b}^{-1}(x, y) & =C^{-1}(x, y) \delta_{a b}+\frac{\lambda}{6 N}\left(\phi_{c} \phi_{c}\right) \delta_{a b} \delta(x-y)+\frac{\lambda}{3 N} \phi_{a} \phi_{b} \delta(x-y), \\
\mathbf{S}_{\mathrm{int}}[\phi, \varphi] & =\int_{x}\left(\frac{\lambda}{6 N} \phi_{a} \varphi_{a} \varphi_{b} \varphi_{b}+\frac{\lambda}{4 ! N}\left(\varphi_{a} \varphi_{a}\right)^{2}\right) .
\end{aligned}
$$

In order to construct the $1 / N$ expansion, one should take into account the implicit $N$-dependence due to the presence of $N$ variables. This is done by counting any "singletrace" invariant as contributing with a factor $N$. There are two types of such invariants in the $O(N)$ model: $\operatorname{Tr}\left[G^{n}\right]$ and $\phi_{a}\left(G^{n}\right)_{a b} \phi_{b}$. Taking into account also the explicit factor $N^{-1}$ in the coupling, one immediately finds that the first three terms in:

$$
\boldsymbol{\Gamma}[\phi, G]=\mathbf{S}[\phi]+\frac{1}{2} \operatorname{Tr}\left[\ln G^{-1}\right]+\frac{1}{2} \operatorname{Tr}\left[G_{0}^{-1} G\right]+\boldsymbol{\Gamma}_{2}[\phi, G],
$$

all scale like $N$, except the contribution from the last term in (2.32) which is of order one. Next, one observes that the last term can be expanded as:

$$
\boldsymbol{\Gamma}_{2}[\phi, G]=\boldsymbol{\Gamma}_{2}^{(1)}[\phi, G]+\boldsymbol{\Gamma}_{2}^{(0)}[\phi, G]+\boldsymbol{\Gamma}_{2}^{(-1)}[\phi, G]+\ldots, \quad \text { with } \boldsymbol{\Gamma}_{2}^{(p)}[\phi, G] \sim N^{p}
$$

This is again somewhat non trivial. We first review the Feynman expansion of $\boldsymbol{\Gamma}_{2}[\phi, G]$ for the $O(N)$ model. In (2.33) there are two kinds of vertices, a trivalent and a tetravalent one, which we represent in figure 1 . The solid lines track the identification of the indices in the vertex. The dashed edge symbolizes the vertex (in an intermediate field representation it would correspond to the propagator of the intermediate field), and the blue dotted halfedge represents the background field $\phi$.

The vertices are connected by propagators $G$ which connect the solid half edges into solid edges. An example of a Feynman graph is presented in figure 2.

We notice first of all that $\boldsymbol{\Gamma}_{2}[\phi, G]$, and hence $\boldsymbol{\Gamma}[\phi, G]$, will only contain even powers of $\phi$. This is obvious at one-loop level, because there are only even powers in the action (2.31), and of course in the second variation (2.32). And is also true beyond one loop, because a single $\phi$ is attached to each 3 -valent vertex, while 4 -valent vertices carry 


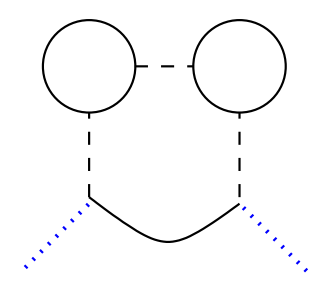

Figure 2. An example of a graph with one tetravalent and two trivalent vertices.

no powers of $\phi$. Therefore, any closed graph will necessarily contain an even number of $\phi$ fields. As a consequence, the equation of motion $\delta \boldsymbol{\Gamma} / \delta \phi=0$ admits the solution $\phi=0$, which is the only solution giving an invariant 1-point function. Whether such a solution is stable or not, and whether there are other stable solutions, will depend on the space dimension. In particular, in $d \leq 2$ spontaneous symmetry breaking of continuous symmetries is impossible $[60,61]$, hence we do not expect other stable solutions for $\phi{ }^{6}$

Coming back to the $1 / N$ expansion, from the Feynman rules one obtains a trace over the vector indices of $G$ to some power for each closed loop of the solid strands, hence each such loop should be counted as a factor $N$ (as seen for example by taking $G_{a b} \sim \delta_{a b}$ ). Each vertex brings instead a factor $1 / N$. The open strands connect pairwise the background fields $\phi$, and correspond to scalar product of the type $\phi_{a}\left(G^{n}\right)_{a b} \phi_{b}$, so they also should be counted as a factor $N$ (as seen for example by taking $\phi_{a} \phi_{b} \sim G_{a b} \sim \delta_{a b}$ ).

The power counting in $N$ is transparent in a loop vertex representation [63] (or cacti representation) in intermediate field. The loops of vector indices are contracted into loop vertices (of arbitrary degree) and the original Feynman vertices become edges of the intermediate field (the black dashed edges in figure 2). The open strands can be contracted to external vertices (of degree two). In this representation the scaling with $N$ of a graph is $N^{-E+L+L_{\text {ext }}}$ where $E$ is the number of intermediate field edges (i.e. vertices in the original Feynman representation), $L$ the number of loop vertices, and $L_{\text {ext }}$ the number of external vertices (i.e. half the number of background fields). As the graph is connected, the number of excess edges ${ }^{7}$ in the intermediate field is $E-\left(L+L_{\text {ext }}\right)+1=\omega \geq 0$.

It follows that the scaling in $N$ of a graph is $N^{-\omega+1}$, hence the graphs contributing to $\boldsymbol{\Gamma}_{2}$ scale at most like $N$ and they scale like $N$ only if they are trees in the intermediate field. Furthermore, the graphs contributing to $\boldsymbol{\Gamma}_{2}[\phi, G]$ must at the same time be $2 \mathrm{PI}$ from the point of view of the original propagators, which translates into the constraint that the tree has no vertices of degree greater than one. Thus only one graph (the double tadpole of figure 3) contributes at leading order (LO):

$$
\Gamma_{2}^{(1)}[\phi, G]=\frac{\lambda}{4 ! N} \int_{x} G_{a a}(x, x) G_{b b}(x, x)=N \frac{\lambda}{4 !} \int_{x} G(x, x)^{2},
$$

which in particular is independent of $\phi$. In the last expression in order to make the $N$ -

\footnotetext{
${ }^{6}$ However, one should keep in mind that the large- $N$ limit can sometimes lead to an apparently opposite conclusion, as explained for the chiral Gross-Neveu model by Witten [62]. See also [29] for an analogue phenomenon in a tensor-valued version of the Gross-Neveu model.

${ }^{7}$ Loops (in the physics literature) for the intermediate field.
} 


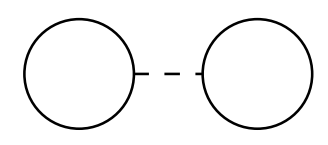

Figure 3. The leading order contribution of the large- $N$ expansion in the $O(N)$ model.

dependence more explicit we restricted to $G_{a b}(x, y)=G(x, y) \delta_{a b}$, which is valid on shell in the symmetric phase.

The equations of motion of $\boldsymbol{\Gamma}[\phi, G]$ at LO read:

$$
\begin{aligned}
0 & =\left(C^{-1} \phi_{a}\right)(x)+\frac{\lambda}{6 N} \phi_{a}(x)\left(\phi_{b}(x) \phi_{b}(x)\right)+\frac{\lambda}{6 N} \phi_{a}(x) G_{b b}(x, x), \\
G_{a b}^{-1}(x, y) & =\left(C^{-1}(x, y)+\frac{\lambda}{6 N} G_{c c}(x, x) \delta(x-y)+\frac{\lambda}{6 N}\left(\phi_{c} \phi_{c}\right) \delta(x-y)\right) \delta_{a b} .
\end{aligned}
$$

The first one clearly admits the $\phi_{a}=0$ solution, which plugged back into the second equation leads to the large- $N$ Schwinger-Dyson (SD) equations for the 2-point function: ${ }^{8}$

$$
G_{a b}^{-1}(x, y)=\left(C^{-1}(x, y)+\frac{\lambda}{6 N} G_{c c}(x, x) \delta(x-y)\right) \delta_{a b} .
$$

As anticipated, we find that $G_{a b}=G(x, y) \delta_{a b}$ on shell, with the scalar part satisfying $G^{-1}(x, y)=C^{-1}(x, y)+\frac{\lambda}{6} G(x, x) \delta(x-y)$. Notice also that a nonzero solution for $\phi_{a}$ (necessarily a constant solution because of translation invariance) implies that it is a zero mode of the inverse 2-point function, i.e. $\left(G_{a b}^{-1} \phi_{b}\right)(x)=0$ : this is because in the case of spontaneous symmetry breaking we have $N-1$ Goldstone modes and only one radial mode, but by keeping only LO terms we have discarded the latter. Including subleading terms it is no longer true that the equation of motion of $\phi$ can be written as $\left(G_{a b}^{-1} \phi_{b}\right)(x)=0$ : in particular in the LO approximation we have discarded a $\frac{\lambda}{3 N} G_{a b}(x, x) \phi_{b}$ term in $(2.37)$ and a $\frac{\lambda}{3 N} \phi_{a} \phi_{b} \delta(x-y)$ term in (2.38), both coming from the one-loop part of the 2PI effective action.

At next-to-leading order (NLO), and for $\phi=0$, we have graphs with $E=L$, always with the 2PI restriction: they are the closed chains of bubbles depicted in figure 4 . The form an infinite family, but thanks to their simple structure they can be summed. In fact, by introducing the kernel

$$
\mathcal{K}(x, y)=\frac{\lambda}{6 N} G_{a b}(x, y) G_{b a}(x, y)=\frac{\lambda}{6} G(x, y)^{2},
$$

we find

$$
\boldsymbol{\Gamma}_{2}^{(0)}[\phi, G]=\sum_{n \geq 1} \frac{(-1)^{n+1}}{2 n} \operatorname{Tr}\left[\mathcal{K}^{n}\right]=\frac{1}{2} \operatorname{Tr}[\ln (\mathbf{1}+\mathcal{K})] .
$$

Still at NLO, but for $\phi \neq 0$, we also have graphs like those of figure 4 but with exactly one solid propagator line being replaced by a background field insertion at each of its end

\footnotetext{
${ }^{8}$ We notice that in $d=0$ (and fixing for example $C=1$ ) the SD equation becomes a simple quadratic equation for $G$ with solution $G=3(-1 \pm \sqrt{1+2 \lambda / 3}) / \lambda$, thus exhibiting a well-known singularity at a negative value of the coupling (e.g. [64]). For $d \geq 1$ instead (with $C^{-1}=-\partial^{2}+m^{2}$ ), the SD equation simply leads to a renormalization of the mass (a finite one in $d=1$ ).
} 


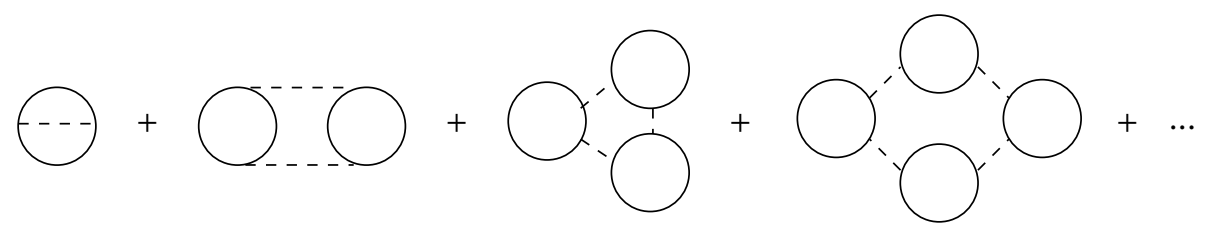

Figure 4. The next-to-leading order contribution of the large- $N$ expansion in the $O(N)$ model.

vertices (e.g. the graph in figure 2; see [52] for more details). Such graphs lead again to an action term which is quadratic in $\phi$, thus not affecting the existence of the $\phi=0$ solution.

\subsection{Large- $N$ expansion as the loop expansion of an auxiliary theory}

Notice that (2.41) looks like the result we would obtain from a standard Gaussian integral with inverse covariance $\mathbf{1}+\mathcal{K}$. It turns out that the large- $N$ expansion for the $2 \mathrm{PI}$ effective action of the vector model can indeed be cast as a loop expansion for an auxiliary bilocal theory, as we are now going to show.

Consider the partition function for the vector model, which corresponds to

$$
Z=e^{\mathbf{W}[0,0]}=e^{-\boldsymbol{\Gamma}[\phi, \underline{G}]}=\int[d \varphi] e^{-\mathbf{S}[\varphi]},
$$

with the action (2.31). Next, insert in the functional integral the identity:

$$
\begin{aligned}
1 & =\int[d \tilde{G}] \delta\left(N \tilde{G}(x, y)-\varphi_{a}(x) \varphi_{a}(y)\right) \\
& =\int[d \tilde{G}][d \tilde{\Sigma}] e^{-\frac{1}{2} \int_{x, y} \tilde{\Sigma}(x, y)\left(N \tilde{G}(x, y)-\sum_{a} \varphi_{a}(x) \varphi_{a}(y)\right)}
\end{aligned}
$$

exploit the delta function to write the interaction in terms of $\tilde{G}$, and then perform the integral over $\varphi$ :

$$
\begin{aligned}
Z & =\int[d \varphi][d \tilde{G}][d \tilde{\Sigma}] e^{-\mathbf{S}[\varphi]-\frac{1}{2} \int_{x, y} \tilde{\Sigma}(x, y)\left(N \tilde{G}(x, y)-\sum_{a} \varphi_{a}(x) \varphi_{a}(y)\right)} \\
& =\int[d \tilde{G}][d \tilde{\Sigma}] e^{-N\left\{\frac{1}{2} \operatorname{Tr}\left[\left(C^{-1}-\tilde{\Sigma}\right) \tilde{G}\right]+\frac{1}{2} \operatorname{Tr}[\ln (\tilde{\Sigma})]+\frac{\lambda}{4 !} \int_{x} \tilde{G}(x, x)^{2}\right\}} \\
& \equiv \int[d \tilde{G}][d \tilde{\Sigma}] e^{-N \mathbf{S}_{\mathrm{eff}}[\tilde{G}, \tilde{\Sigma}]}
\end{aligned}
$$

We have thus rewritten the original functional integral over $N$ (local) variables as an integral over just two (bilocal) variables, and all the dependence on $N$ is now explicit and factored in front of the total action. Therefore, the $1 / N$ expansion takes the standard form of a loop (i.e. saddle-point) expansion. We shift the fields to the saddle point value: $\tilde{G}(x, y)=\underline{G}(x, y)+N^{-1 / 2} g(x, y), \tilde{\Sigma}(x, y)=\underline{\Sigma}(x, y)+N^{-1 / 2} \sigma(x, y)$. Expanding to second order in $g$ and $\sigma$, we find:

$$
Z \simeq e^{-N \mathbf{S}_{\mathrm{eff}}[\underline{G}, \underline{\Sigma}]} \int[d g][d \sigma] e^{-\mathbf{S}_{\mathrm{eff}}^{(2)}[\underline{G}, \underline{\Sigma} ; g, \sigma]}
$$


where the on-shell effective classical action coincides with the on-shell 2PI effective action at LO:

$$
\mathbf{S}_{\mathrm{eff}}[\underline{G}, \underline{\Sigma}]=\frac{1}{2} \operatorname{Tr}\left[\ln \underline{G}^{-1}\right]+\frac{1}{2} \operatorname{Tr}\left[C^{-1} \underline{G}\right]+\frac{\lambda}{4 !} \int_{x} \underline{G}(x, x)^{2} .
$$

We have also defined the quadratic part of the action:

$$
\begin{aligned}
\mathbf{S}_{\text {eff }}^{(2)}[\underline{G}, \underline{\Sigma} ; g, \sigma]= & -\frac{1}{4} \int_{x_{1}, x_{2}, x_{3}, x_{4}} \sigma\left(x_{1}, x_{2}\right) \underline{\mathcal{K}}_{4}\left(x_{1}, x_{2} ; x_{3}, x_{4}\right) \sigma\left(x_{3}, x_{4}\right) \\
& -\frac{1}{2} \int_{x_{1}, x_{2}} \sigma\left(x_{1}, x_{2}\right) g\left(x_{1}, x_{2}\right)+\frac{\lambda}{4 !} \int_{x} g(x, x)^{2},
\end{aligned}
$$

where the kernel is:

$$
\underline{\mathcal{K}}_{4}\left(x_{1}, x_{2} ; x_{3}, x_{4}\right)=\frac{1}{2}\left(\underline{G}\left(x_{1}, x_{3}\right) \underline{G}\left(x_{2}, x_{4}\right)+\underline{G}\left(x_{1}, x_{4}\right) \underline{G}\left(x_{2}, x_{3}\right)\right) .
$$

Performing the Gaussian integrals we find:

$$
\begin{aligned}
Z & \simeq \frac{e^{-N \mathbf{S}_{\text {eff }}[\underline{G}, \underline{\Sigma}]}}{\left(\operatorname{det}\left(\underline{\mathcal{K}}_{4}\right)\right)^{1 / 2}} \int[d g] e^{-\frac{1}{4} \int_{x_{1}, x_{2}, x_{3}, x_{4}} g\left(x_{1}, x_{2}\right) \underline{\mathcal{K}}_{4}^{-1}\left(x_{1}, x_{2} ; x_{3}, x_{4}\right) g\left(x_{3}, x_{4}\right)-\frac{\lambda}{4 !} \int_{x} g(x, x)^{2}} \\
& =e^{-N \mathbf{S}_{\text {eff }}[\underline{G}]-\frac{1}{2} \operatorname{Tr}[\ln (1+\underline{\mathcal{K}})]}
\end{aligned}
$$

where the kernel $\underline{\mathcal{K}}$ in the final result is exactly the one in (2.40) evaluated on shell. Notice that due to the interaction being local rather than bilocal (compare with the SYK model in the next section), the 4 -point kernel $\underline{\mathcal{K}}_{4}$ reduces to the 2 -point kernel $\underline{\mathcal{K}}$. We have thus recovered the LO and NLO of $\boldsymbol{\Gamma}[\underline{\phi}, \underline{G}]$ by a standard saddle-point method.

A remark is in order. In $\boldsymbol{\Gamma}[\bar{\phi}, \underline{G}]$ the on-shell fields should be obtained from the full effective action. As we explained, $\underline{\phi}=0$ is valid to all orders in the symmetric phase, but the on-shell value $\underline{G}$ receives corrections in $1 / N$. Expanding $\boldsymbol{\Gamma}[0, G] \simeq N \boldsymbol{\Gamma}^{(1)}[0, G]+\boldsymbol{\Gamma}^{(0)}[0, G]$, we find an expansion for the solution $\underline{G}=\underline{G}^{(0)}+N^{-1} \underline{G}^{(-1)}$, and therefore, $\boldsymbol{\Gamma}[\phi, \underline{G}]=$ $N \boldsymbol{\Gamma}^{(1)}\left[0, \underline{G}^{(0)}\right]+\boldsymbol{\Gamma}^{(0)}\left[0, \underline{G}^{(0)}\right]+O\left(N^{-1}\right)$, because $\frac{\delta \boldsymbol{\Gamma}^{(1)}}{\delta G}\left[0, \underline{G}^{(0)}\right]=0$ by construction.

\section{$3 \quad 2$ PI effective action for the SYK model}

The SYK model is defined in terms of $N$ Majorana fermions in one dimension, with anti commutation relation $\left\{\psi_{a}, \psi_{b}\right\}=\delta_{a b}$, and with action

$$
\mathbf{S}_{\mathrm{SYK}}[\psi]=\int d t\left(\frac{1}{2} \psi_{a} \partial_{t} \psi_{a}+\frac{\mathrm{i}^{q / 2}}{q !} J_{a_{1} \ldots a_{q}} \psi_{a_{1}} \ldots \psi_{a_{q}}\right) .
$$

Here, $J_{a_{1} \ldots a_{q}}$ is a random totally antisymmetric tensorial coupling, with Gaussian distribution

$$
\left.P\left[J_{a_{1} \ldots a_{q}}\right] \propto \exp \left\{-\frac{N^{q-1}\left(J_{a_{1} \ldots a_{q}}\right)^{2}}{2(q-1) ! J^{2}}\right\} \quad \text { (no sum }\right) .
$$

We will denote with a bar the average over the disorder:

$$
\overline{A[J]}=\int\left(\prod_{a_{1}<a_{2}<\ldots<a_{q}}\left[d J_{a_{1} \ldots a_{q}}\right] P\left[J_{a_{1} \ldots a_{q}}\right]\right) A[J] .
$$


For example, we have

$$
\overline{J_{a_{1} \ldots a_{q}} J_{b_{1} \ldots b_{q}}}=\frac{q !(q-1) !}{N^{q-1}} J^{2} \Pi_{a_{1} \ldots a_{q}, b_{1} \ldots b_{q}},
$$

where $\prod_{a_{1} \ldots a_{q}, b_{1} \ldots b_{q}}$ is the projector on antisymmetric rank- $q$ tensors:

$$
\Pi_{a_{1} \ldots a_{q}, b_{1} \ldots b_{q}}=\frac{1}{q !} \sum_{\sigma \in \mathfrak{S}_{q}} \epsilon(\sigma) \prod_{i=1}^{q} \delta_{a_{i} b_{\sigma(i)}},
$$

with $\mathfrak{S}_{q}$ the symmetric group on $q$ elements, and $\epsilon(\sigma)$ the sign of the permutation $\sigma$.

One deals with the randomness of the coupling by computing quenched averages of intensive quantities, such as the free energy or the entropy, which in general (e.g. for models with short-range interactions) are self-averaging, i.e. in the thermodynamic limit they converge with probability one to their average. In particular, the quenched free energy is

$$
-N \bar{F}=\overline{\ln Z}=\int\left(\prod_{a_{1}<a_{2}<\ldots<a_{q}}\left[d J_{a_{1} \ldots a_{q}}\right] P\left[J_{a_{1} \ldots a_{q}}\right]\right) \ln \int[d \psi] e^{-\mathbf{S}_{\mathrm{SYK}}[\psi]} .
$$

The expansion in Feynman graphs is standard, with the only peculiarity that each vertex carries a tensor $J_{a_{1} \ldots a_{q}}$ with each index being associated to one half-edge.

In the same way as we defined a quenched free energy, we can define the quenched generating functionals of connected, 1PI, and 2PI diagrams, by constructing them in the usual way for each realization of the disorder and taking the average over disorder at the end. One should be careful with defining the generating functionals in such a way, because for example the averaging procedure does not in general commute with evaluating the effective action on shell. However, for the SYK model it can be shown by an analysis of the diagrams that commutativity holds at LO and NLO, a fact that here we will only show a posteriori by comparison to known results. ${ }^{9}$

We can therefore repeat all the construction of the 2PI effective action as above, with the novel feature that 2PI graphs contributing to $\boldsymbol{\Gamma}_{2}$ now have to be averaged over disorder, and that the fermionic nature of the model brings in some minus factors. We have

$$
\boldsymbol{\Gamma}[\Psi, G]=\mathbf{S}_{\mathrm{SYK}}[\Psi]-\frac{1}{2} \operatorname{Tr}\left[\ln G^{-1}\right]-\frac{1}{2} \operatorname{Tr}\left[G_{0}^{-1} G\right]+\boldsymbol{\Gamma}_{2}[\Psi, G] .
$$

In order to simplify the analysis of the large- $N$ limit we directly set $\Psi=0$, which is again justified by the absence of spontaneous symmetry breaking. By the same reason we could also fix $G_{a b}(x, y)=\delta_{a b} G(x, y)$, although in general it will be more transparent to keep the general expression. After averaging over the disorder all the diagrams lead to

\footnotetext{
${ }^{9}$ Note that in the standard way of obtaining LO and NLO results for the SYK model a replica diagonal ansatz is taken for the bilocal field, which is justified by the fact that for the SYK model quenched and annealed averages coincide at LO and NLO [7, 18]. At NNLO, within the replica method one should take into account interactions between different replicas (i.e. off-diagonal fluctuations of the bilocal field), while in the 2PI formalism one should take into account diagrams that arise when the averaging is done after the on-shell evaluation.
} 


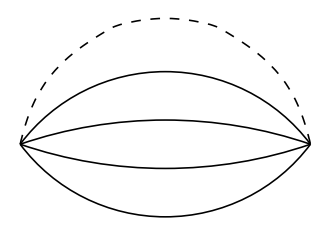

Figure 5. The fundamental melon for $q=4$. The dashed line represents the Wick contraction associated to the quenched average.

different multiple traces of powers of $G_{a b}\left(t, t^{\prime}\right)$, and as before we should count each trace as contributing a factor $N$. We find in this way an expansion of the same type as (2.35). Remembering that in the large- $N$ limit the disorder average selects melons [3], we find that $\boldsymbol{\Gamma}_{2}[0, G]$ at leading order in $1 / N$ is given by the fundamental vacuum melon of figure 5 , which is the only $2 \mathrm{PI}$ melon graph, with propagators given by $G$, i.e.:

$$
\begin{aligned}
\Gamma_{2}^{(1)}[0, G] & =-\frac{1}{2 q !} \overline{J_{a_{1} \ldots a_{q}} J_{b_{1} \ldots b_{q}}} \int_{t, t^{\prime}} \prod_{c=1}^{q} G_{a_{c} b_{c}}\left(t, t^{\prime}\right) \\
& =-\frac{J^{2}}{2 q N^{q-1}} \int_{t, t^{\prime}} G_{a a}\left(t, t^{\prime}\right)^{q}=-\frac{J^{2} N}{2 q} \int_{t, t^{\prime}} G\left(t, t^{\prime}\right)^{q} .
\end{aligned}
$$

Notice that having chosen a Wick pairing of fermions to give the propagators (in $q$ ! ways, thus canceling one of the $1 / q$ ! factors that come from the vertices), the average over disorder produces many different types of contractions, due to the projector in (3.4), but in the second line we have taken the only contraction that contributes at LO. The number of traces (and hence the power of $N$ ) is in general given by the number $c(\sigma)$ of cycles in the disjoint cycle decomposition of the permutation $\sigma$ appearing in the projector. Thus permutations that can be obtained with a single transposition contribute to the NLO:

$$
\begin{aligned}
& \left(-\frac{1}{2 q !} \overline{J_{a_{1} \ldots a_{q}} J_{b_{1} \ldots b_{q}}} \int_{t, t^{\prime}} \prod_{c=1}^{q} G_{a_{c} b_{c}}\left(t, t^{\prime}\right)\right)_{\mathrm{NLO}} \\
& =-\frac{J^{2}}{2 q N^{q-1}}\left(\begin{array}{l}
q \\
2
\end{array}\right) \int_{t, t^{\prime}} G_{a a}\left(t, t^{\prime}\right)^{q-2} G_{b c}\left(t, t^{\prime}\right) G_{c b}\left(t, t^{\prime}\right)=-\frac{J^{2}(q-1)}{4} \int_{t, t^{\prime}} G\left(t, t^{\prime}\right)^{q} .
\end{aligned}
$$

The full $2 \mathrm{PI}$ effective action at leading order in $1 / N$ is thus:

$$
\frac{1}{N} \boldsymbol{\Gamma}[0, G]=-\frac{1}{2} \operatorname{Tr}\left[\ln G^{-1}\right]-\frac{1}{2} \operatorname{Tr}\left[\partial_{t} G\left(t, t^{\prime}\right)\right]-\frac{J^{2}}{2 q} \int_{t, t^{\prime}} G\left(t, t^{\prime}\right)^{q},
$$

which coincides with the bilocal action derived in [9] by a change of variables within the replica method. The equivalence with [9] implies in particular that the equations of motion are the same and coincide with the SD equations: ${ }^{10}$

$$
G^{-1}\left(t, t^{\prime}\right)=\partial_{t} \delta\left(t, t^{\prime}\right)-J^{2} G\left(t, t^{\prime}\right)^{q-1},
$$

and that the Schwarzian action controlling the conformal fluctuations can be derived in a similar fashion as in that paper. We denote the solution of (3.11) as $\underline{G}\left(t, t^{\prime}\right)$.

\footnotetext{
${ }^{10}$ Here one should remember that for Majorana fermions $G\left(t, t^{\prime}\right)=-G\left(t^{\prime}, t\right)$.
} 
Recalling eq. (2.14) and (2.18), the second derivative $\boldsymbol{\Gamma}_{G G}[0, \underline{G}]$ is equal to the inverse of $\mathcal{F}\left(t_{1}, t_{2}, t_{3}, t_{4}\right)$, i.e. of the full 4 -point function minus the disconnected channel $(1 \rightarrow 2,3 \rightarrow$ 4). Interestingly, such channel is the leading-order (and uninteresting) term in the SYK 4-point function $[3,5] .{ }^{11}$ Therefore, $\boldsymbol{\Gamma}_{G G}[0, \underline{G}]$ captures precisely the inverse of the object of interest in SYK. We can compute this from our LO effective action, and recover the corresponding result of the SYK 4-point function given by the sum of the ladder diagrams, see [3-5]. Taking into account that:

$$
\frac{\delta G_{34}}{\delta G_{12}}=\frac{1}{2}\left(\delta\left(t_{1}-t_{3}\right) \delta\left(t_{2}-t_{4}\right)-\delta\left(t_{1}-t_{4}\right) \delta\left(t_{2}-t_{3}\right)\right) \equiv I_{-}\left(t_{1}, t_{2} ; t_{3}, t_{4}\right),
$$

with $I$ the orthogonal projector on antisymmetric functions, and denoting the on-shell four point kernel:

$$
\underline{\mathcal{K}}\left(t_{1}, t_{2} ; t_{3}, t_{4}\right)=-J^{2}(q-1) \underline{G}\left(t_{1}, t_{3}\right) \underline{G}\left(t_{2}, t_{4}\right) \underline{G}\left(t_{3}, t_{4}\right)^{q-2},
$$

we get:

$$
\begin{aligned}
\boldsymbol{\Gamma}_{G_{34}}= & \frac{1}{2} \underline{G}^{-1}\left(t_{4}, t_{3}\right)+\frac{1}{2} \partial_{t} \delta\left(t_{3}-t_{4}\right)-\frac{1}{2} J^{2}\left[\underline{G}\left(t_{3}, t_{4}\right)\right]^{q-1} \\
\boldsymbol{\Gamma}_{G_{12} G_{34}}= & -\frac{1}{4} \underline{G}^{-1}\left(t_{4}, t_{1}\right) \underline{G}^{-1}\left(t_{2}, t_{3}\right)+\frac{1}{4} \underline{G}^{-1}\left(t_{4}, t_{2}\right) \underline{G}^{-1}\left(t_{1}, t_{3}\right) \\
& +\frac{1}{4}\left[\delta\left(t_{1}-t_{3}\right) \delta\left(t_{2}-t_{4}\right)-\delta\left(t_{1}-t_{4}\right) \delta\left(t_{2}-t_{3}\right)\right]\left[-J^{2}(q-1) \underline{G}\left(t_{3}, t_{4}\right)^{q-2}\right] \\
= & -\frac{1}{2} \int_{t, t^{\prime}} \underline{G}^{-1}\left(t_{1}, t\right) \underline{G}^{-1}\left(t_{2}, t^{\prime}\right)\left[I_{-}(1-\underline{\mathcal{K}})\right]\left(t, t^{\prime} ; t_{3}, t_{4}\right) .
\end{aligned}
$$

Inverting the last expression we find:

$$
\mathcal{F}\left(t_{1}, t_{2}, t_{3}, t_{4}\right)=\int_{t, t^{\prime}}\left(\frac{1}{1-\underline{\mathcal{K}}}\right)\left(t_{1}, t_{2}, t, t^{\prime}\right)\left(-\underline{G}\left(t, t_{3}\right) \underline{G}\left(t^{\prime}, t_{4}\right)+\underline{G}\left(t, t_{4}\right) \underline{G}\left(t^{\prime}, t_{3}\right)\right),
$$

which is precisely the starting point of the computations in $[3-5]$.

\subsection{Next-to-leading order action}

As in the vector model of the previous section, (3.10) will receive corrections at higher orders in $1 / N$. We want to show that the NLO correction can be interpreted as the result of performing the Gaussian integral over the fluctuations in the usual bilocal action expanded to quadratic order. In order to do that, we need to understand which 2PI diagrams contribute at NLO, a question that has been addressed in detail in [40] for the colored version of the model, which is a special case of the generalization of the SYK model introduced by Gross and Rosenhaus [6] (see also [50] for a discussion of the same

${ }^{11}$ As a reminder, the 4 -point function we are talking about is:

$$
\frac{1}{N^{2}}\left\langle\psi_{m}\left(t_{1}\right) \psi_{m}\left(t_{2}\right) \psi_{n}\left(t_{3}\right) \psi_{n}\left(t_{4}\right)\right\rangle=G\left(t_{12}\right) G\left(t_{34}\right)+\frac{1}{N} \mathcal{F}^{L O}\left(t_{1}, t_{2}, t_{3}, t_{4}\right)+\ldots
$$

The $G\left(t_{12}\right) G\left(t_{34}\right)$ part is precisely the channel missing when taking the derivatives as in (2.14), and therefore, evaluating this derivative at $\mathrm{LO}$ will give us $\mathcal{F}^{L O}$. The latter was computed in [3-5]. 

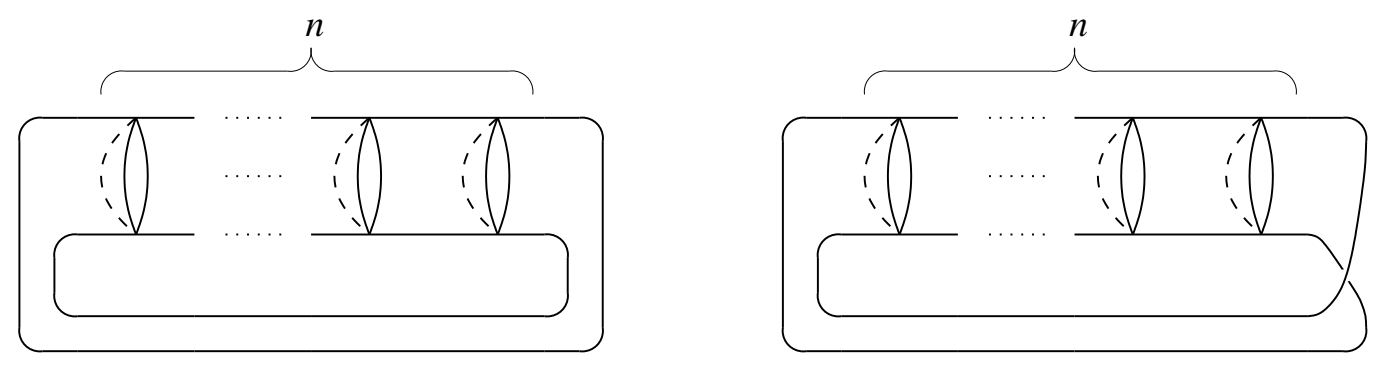

Figure 6. NLO contributions with $n$ rungs, without (left) and with (right) twist.

model at NLO). It turns out that similar type of diagrams dominate also the standard SYK model, but have to be accompanied by the twisted melons (3.9), which are absent in the colored case.

The NLO 2PI vacuum graphs are thus given by all the periodic ladders with $n \geq 1$ rungs, with or without one twist of the rails, see figure 6 . One should notice that the case $n=1$ is quite special. First, the case $n=1$ without twist is 2-particle reducible if $q=4$, but since it evaluates to zero for any $q$ (because $G(t, t)=0$ due to the fermions' anti commutation), we can formally include it in the list. On the other hand, the case $n=1$ with twist corresponds again to a fundamental melon, thus one might think that it is LO rather than NLO. However, this corresponds precisely to the twisted melons in (3.9), which therefore can be conveniently grouped with the ladders. Although such ladders form an infinite family of graphs, they can be summed in a similar way as to what we did for the vector case, i.e. by introducing a kernel for the insertion of a rung. One important difference is that now the kernel carries two vertices rather than one, which counts for different combinatorial factors and minus signs in the summation (notice that the kernel below has itself another minus sign, due to the fermionic nature of the theory). More explicitly, we have

$$
\Gamma_{2}^{(0)}[0, G]=-\frac{1}{2} \sum_{n \geq 0} \frac{1}{n} \operatorname{Tr}\left[\mathcal{K}^{n} I_{-}\right]=\frac{1}{2} \operatorname{Tr}\left[\ln \left(I_{-}-\mathcal{K} I_{-}\right)\right]
$$

where $I_{-}$and $\mathcal{K}$ are given in (3.13) and (3.14) (now off-shell), and to obtain the last equality we used the fact that $I_{-}=I_{-}^{n}$ because it is a projector, and $\left[\mathcal{K}, I_{-}\right]=0$. Evaluating $\boldsymbol{\Gamma}_{2}^{(0)}[0, G]$ on the solution of the LO equations of motion, we find that this is the same result that one would obtain by integrating the quadratic fluctuations of the bilocal effective action of $[5,7]$.

In order to see that, we just have to repeat what we have done for the vector case, with the important difference that due to the disorder one has to use the replica method. The quenched average in the SYK model can be performed using the replica method, at the cost of introducing $n$ replicas of the system, and having to take the non-trivial limit $n \rightarrow 0$, which is needed in order to evaluate the quenched free energy:

$$
\overline{\ln Z}=\lim _{n \rightarrow 0} \partial_{n} \overline{Z^{n}}
$$


One finds [7]:

$$
\overline{Z^{n}}=\int\left(\prod_{\alpha \beta}\left[d G^{\alpha \beta}\right]\left[d \Sigma^{\alpha \beta}\right]\right) e^{-N \mathbf{S}_{\mathrm{eff}}[G, \Sigma]},
$$

where:

$$
\mathbf{S}_{\mathrm{eff}}[G, \Sigma]=-\frac{1}{2} \widehat{\operatorname{Tr}} \ln \left(\partial_{t}-\Sigma\right)+\frac{1}{2} \sum_{\alpha \beta} \int_{t, t^{\prime}}\left(\Sigma^{\alpha \beta}\left(t, t^{\prime}\right) G^{\alpha \beta}\left(t, t^{\prime}\right)-\frac{J^{2}}{q}\left(G^{\alpha \beta}\left(t, t^{\prime}\right)\right)^{q}\right) .
$$

Notice that the disorder average led to an effective bare action which is bilocal even in the interaction term. Performing the saddle-point approximation with a replica diagonal ansatz $\underline{G}^{\alpha \beta}=\underline{G} \delta^{\alpha \beta}$, which is valid up to NLO in $1 / N[7]$, one arrives at [5]:

$$
\overline{\ln Z}=N\left(\frac{1}{2} \operatorname{Tr}\left[\ln \underline{G}^{-1}\right]+\frac{1}{2} \operatorname{Tr}\left[\partial_{t} \underline{G}\left(t, t^{\prime}\right)\right]+\frac{J^{2}}{2 q} \int_{t, t^{\prime}} \underline{G}\left(t, t^{\prime}\right)^{q}\right)-\frac{1}{2} \operatorname{Tr}\left[\ln \left(I_{-}-\tilde{\mathcal{K}} I_{-}\right)\right],
$$

with

$$
\tilde{\mathcal{K}}\left(t_{1}, t_{2} ; t_{3}, t_{4}\right)=\left|\underline{G}\left(t_{1}, t_{2}\right)\right|^{\frac{q-2}{2}} \underline{\mathcal{K}}\left(t_{1}, t_{2} ; t_{3}, t_{4}\right)\left|\underline{G}\left(t_{1}, t_{2}\right)\right|^{\frac{2-q}{2}} .
$$

Since $\frac{1}{2} \operatorname{Tr}\left[\ln \left(I_{-}-\tilde{\mathcal{K}} I_{-}\right)\right]=\frac{1}{2} \operatorname{Tr}\left[\ln \left(I_{-}-\underline{\mathcal{K}} I_{-}\right)\right]$, we recover our $\boldsymbol{\Gamma}[0, \underline{G}]$ up to NLO, as claimed.

Note that the main difference between (3.21) with replica-diagonal ansatz and (2.44) is that the SYK model the interaction part of $\mathbf{S}_{\text {eff }}$ is bilocal while in the $O(N)$ model it is local. This is reflected in the fact that the associated fluctuation kernel is truly a 4-point kernel in the SYK case while it is a 2-point kernel in the $O(N)$ case. From a graphical point of view the bilocality in the SYK model originates from the fact that the NLO graphs are ladders, while in the vector model they are chains of bubbles.

The replica diagonal ansatz used to derive the result above implies that $\overline{\ln Z}=\ln \bar{Z}$, i.e. that quenched and annealed averages coincide (see [18] for a combinatorial proof at LO). Starting at NNLO [7], the two averaging procedures start to differ, or in other words, the replica-symmetric ansatz becomes inaccurate. From the point of view of the 2PI formalism, the complications at NNLO arise from the non-commutativity of averaging over disorder and going on shell, as we discussed before.

\section{2PI effective action for tensor field theories}

A rank- $r$ tensor-valued real bosonic ${ }^{12}$ field in $d$ space time dimensions is a function $\varphi: \mathbb{R}^{d} \rightarrow$ $\otimes_{i=1}^{r} V_{i}$, where $V_{i}$ is the vector space associated to the fundamental representation of a group $\mathcal{G}_{i}$. In other words, the tensor is postulated to be in the fundamental representation of a group $\mathcal{G}=\prod_{i=1}^{r} \mathcal{G}_{i}$. We denote its components as $\varphi_{a_{1} \ldots a_{r}}(x)$, with $x \in \mathbb{R}^{d}$ and $a_{i}=1 \ldots N_{i}$, where $N_{i}=\operatorname{dim}\left(V_{i}\right)$, hence the group acts by the transformation rule:

$$
\varphi_{a_{1} \ldots a_{r}}(x) \rightarrow\left(\prod_{i=1}^{r} R_{a_{i} b_{i}}^{(i)}\right) \varphi_{b_{1} \ldots b_{r}}(x),
$$

\footnotetext{
${ }^{12}$ In the fermionic case we will denote the field with $\psi$, and in dimensions $d>1$ one should remember also that its components are spinors.
} 
with the matrix $R^{(i)}$ belonging to the fundamental representation of the group $\mathcal{G}_{i}{ }^{13}$ We only consider tensor field theories defined by a classical action which is invariant under the action of $\mathcal{G}$.

In the rest of the paper we will only study few specific models, yet we started this section with a very generic definition to emphasize that the construction of the 2PI effective action can be done in full generality. In fact, it is straightforward to define the $2 \mathrm{PI}$ effective action for tensor-valued field theories in $d$ dimensions applying the construction that we reviewed in section 2: all the equations before section 2.1 are in fact still valid, with the collective index now corresponding to an $r$-uple of indices $\left(a_{1} \ldots a_{r}\right)$ plus the spacetime point. For example, in rank 3 the bilocal field $G_{\mathbf{a b}}$ corresponds to $G_{a_{1} a_{2} a_{3} b_{1} b_{2} b_{3}}(x, y)$, and so on. The presence of several fields (as in the GW model defined below) is also straightforward to take into account: one simply needs to extend further the meaning of the vector label by including a field (or color) index $c=1 \ldots q$. In this case, the discrete part of the collective-index $\mathbf{a}$ can be thought as a vector index with $\mathbf{a}=1 \ldots M$, with $M=q \prod_{i=1}^{r} N_{i}$. The crucial property that characterizes a proper tensor model is the symmetry group: for a vector model the natural symmetry group would be $O(M)$, while for a tensor model this is broken by the choice of interaction down to a smaller group with a natural tensorial interpretation (e.g. $O(M)$ is broken down to $O(N)^{r}$ ).

In the following we will consider only the cases $d=0$ and $d=1$, for two types of models: the Carrozza-Tanasa-Klebanov-Tarnopolsky (CTKT) model [22, 59], for winch $r=3$ and $\mathcal{G}_{i}=O(N)$ for $i=1 \ldots 3$, and the Gurau-Witten $(\mathrm{GW})$ model $[1,19]$, in arbitrary rank $r=q-1$ and with $\mathcal{G}_{i}=O(N)$ for $i=1 \ldots q(q-1) / 2$.

\subsection{The bosonic CTKT model in $d=0$}

The CTKT model in zero dimensions is defined by the action:

$$
\mathbf{S}_{\mathrm{CTKT}}[\varphi]=\frac{1}{2} \varphi_{a b c} \varphi_{a b c}+\frac{\lambda}{4 N^{3 / 2}} \varphi_{a_{1} a_{2} a_{3}} \varphi_{a_{1} b_{2} b_{3}} \varphi_{b_{1} a_{2} b_{3}} \varphi_{b_{1} b_{2} a_{3}}
$$

As standard, we refer to the location of an index as a color, e.g. the indices $a_{1}$ and $b_{1}$ in the action above are of color 1 , and so on.

The perturbative expansion can as usual be represented in a diagrammatic way. Due to the tensor structure, there are different possible representation, which we depict in figure 7 .

The corresponding $2 \mathrm{PI}$ effective action is constructed as explained in section 2, and in particular equation (2.26) is still valid, with $\mathbf{S}[\phi]=\mathbf{S}_{C T K T}[\phi]$,

$$
\begin{aligned}
\left(G_{0}^{-1}\right)_{a_{1} a_{2} a_{3} b_{1} b_{2} b_{3}}= & \delta_{a_{1} b_{1}} \delta_{a_{2} b_{2}} \delta_{a_{3} b_{3}} \\
& +\frac{\lambda}{N^{3 / 2}}\left(\phi_{c_{1} a_{2} a_{3}} \phi_{c_{1} b_{2} b_{3}} \delta_{a_{1} b_{1}}+\phi_{a_{1} c_{2} a_{3}} \phi_{b_{1} c_{2} b_{3}} \delta_{a_{2} b_{2}}+\phi_{a_{1} a_{2} c_{3}} \phi_{b_{1} b_{2} c_{3}} \delta_{a_{3} b_{3}}\right)
\end{aligned}
$$

\footnotetext{
${ }^{13}$ One could also consider tensors in an irreducible tensor representation of a single group, for example symmetric traceless or antisymmetric tensors for the group $\mathcal{G}=O(N)$. Tensor models of this type (for rank $r=3$ ) have recently been proved to admit a large- $N$ expansion [65].
} 

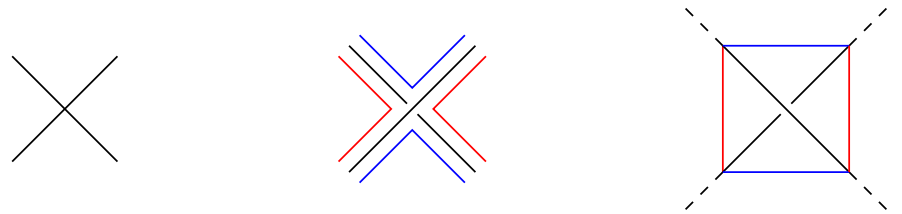

Figure 7. The vertex of the CTKT model (4.2) in three different representation; from left to right: the Feynman representation, the stranded representation, and the "tetrahedron", or edge-colored representation. In the last two the colors track the indices.

and with $\boldsymbol{\Gamma}_{2}[\phi, G]$ constructed from $2 \mathrm{PI}$ graphs with propagator $G_{a_{1} a_{2} a_{3} b_{1} b_{2} b_{3}}$ and interaction

$$
\mathbf{S}_{\mathrm{int}}[\phi, \varphi]=\frac{\lambda}{N^{3 / 2}} \phi_{a_{1} a_{2} a_{3}} \varphi_{a_{1} b_{2} b_{3}} \varphi_{b_{1} a_{2} b_{3}} \varphi_{b_{1} b_{2} a_{3}}+\frac{\lambda}{4 N^{3 / 2}} \varphi_{a_{1} a_{2} a_{3}} \varphi_{a_{1} b_{2} b_{3}} \varphi_{b_{1} a_{2} b_{3}} \varphi_{b_{1} b_{2} a_{3}} .
$$

As in the vector model, $\boldsymbol{\Gamma}_{2}[\phi, G]$, and hence $\boldsymbol{\Gamma}[\phi, G]$, will only contain even powers of $\phi$, and as a consequence, the equation of motion $\delta \boldsymbol{\Gamma} / \delta \phi=0$ admits the solution $\phi=0$, which is the only solution giving an invariant 1-point function. Thus we consider the case of zero background field, $\phi=0$, and study the large- $N$ expansion of $\boldsymbol{\Gamma}_{2}[0, G]$.

In order to do a large- $N$ expansion as in the vector case we need to identify quantities that scale like $N$. In the vector case we saw that $\operatorname{Tr}\left[G^{m}\right] \sim N$ for any $m$. The easiest way to see such scaling is to assume that $G_{a b} \propto \delta_{a b}$ which we know to be true for the on-shell 2-point function. The analogue for the tensor case is to treat any "trace" over a given color as being of order $N$. Again the easiest way to see why it is so is to take $G_{a_{1} a_{2} a_{3} b_{1} b_{2} b_{3}} \propto \delta_{a_{1} b_{1}} \delta_{a_{2} b_{2}} \delta_{a_{3} b_{3}}$, which we know is going to be true on shell, due to the invariance of the theory. The identification of the scaling with $N$ of the graphs contributing to the 2PI effective action is thus reduced to the well-studied problem of identifying the scaling with $N$ of tensor model graphs. We can then borrow the results from [59] and claim that:

- $\boldsymbol{\Gamma}_{2}[0, G]$ can be expanded as:

$$
\boldsymbol{\Gamma}_{2}[0, G]=\sum_{\omega \in \mathbb{N} / 2} \Gamma_{2}^{(3-\omega)}[G], \quad \text { with } \boldsymbol{\Gamma}_{2}^{(p)}[G] \sim N^{p}
$$

- In the large- $N$ limit $\boldsymbol{\Gamma}_{2}[0, G]$ is given by a single diagram, i.e. the fundamental vacuum melon (whose Feynman representation is the same as in figure 5 with no dashed line, and whose tetrahedron representation is given in figure 8), with propagators given by $G$ : since the interaction is the known one, we know that melons dominate the large- $N$ limit, and the fundamental melon is the only $2 \mathrm{PI}$ melon. ${ }^{14}$

\footnotetext{
${ }^{14}$ If we do not set $\phi=0$, we obtain in addition a term of the type $\phi^{2} G^{3}$, corresponding to a melon with two $\phi$ external legs and three internal propagators $G$. These terms should be taken into account when looking at fluctuations around the solution, but at quadratic order the fluctuations of $\phi$ decouple from those of $G$ because $\phi=0$ and the action is at least quadratic in $\phi$.
} 


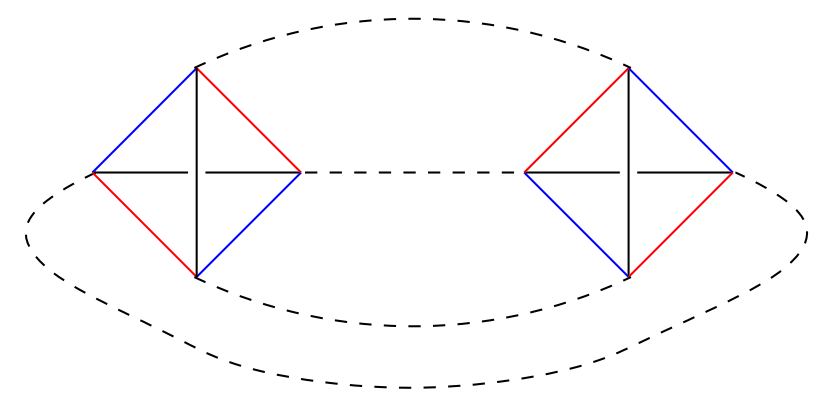

Figure 8. The fundamental melon for the CTKT model in the tetrahedron representation. Dashed edges represent propagators.

Since the fundamental melon diagram comes with a combinatorial factor of 4 , we obtain:

$$
\Gamma_{2}^{(3)}[G]=-\frac{\lambda^{2}}{8 N^{3}} G_{a_{1} a_{2} a_{3} b_{1} b_{2} b_{3}} G_{a_{1} a_{2}^{\prime} a_{3}^{\prime} b_{1} b_{2}^{\prime} b_{3}^{\prime}} G_{a_{1}^{\prime} a_{2} a_{3}^{\prime} b_{1}^{\prime} b_{2} b_{3}^{\prime}} G_{a_{1}^{\prime} a_{2}^{\prime} a_{3} b_{1}^{\prime} b_{2}^{\prime} b_{3}}
$$

In the symmetric phase, the two point function is diagonal in the tensor indices:

$$
G_{a_{1} a_{2} a_{3} b_{1} b_{2} b_{3}}=G \delta_{a_{1} b_{1}} \delta_{a_{2} b_{2}} \delta_{a_{3} b_{3}}
$$

hence:

$$
\Gamma_{2}^{(3)}[G]=-\frac{1}{8} \lambda^{2} N^{3} G^{4}
$$

and we obtain at leading order in $1 / N$ :

$$
\frac{1}{N^{3}} \boldsymbol{\Gamma}[0, G]=\frac{1}{2} \ln G^{-1}+\frac{1}{2} G-\frac{1}{8} \lambda^{2} G^{4} .
$$

The LO equations of motion are simply:

$$
G^{-1}=1-\lambda^{2} G^{3}
$$

which we recognize as the SD equations at leading order in the $1 / N$ expansion [59].

Following [59], one finds that at next-to-leading order the dominant graphs are generated by inserting melonic 2-point functions in the propagators of the three core graphs obtained form the one depicted in figure 9 by permutation of the colors. Since any insertion of a melonic 2-point function makes the graph 2-particle reducible, we conclude that at NLO there is only a finite number of $2 \mathrm{PI}$ graphs, i.e. the three core graphs themselves. They correspond to three contractions like:

$$
\frac{\lambda}{4 N^{3 / 2}} G_{a_{1} a_{2} a_{3} a_{1} b_{2} b_{3}} G_{a_{1}^{\prime} a_{2} a_{3} a_{1}^{\prime} b_{2} b_{3}} .
$$

With the diagonal ansatz for the two point function we obtain:

$$
\frac{1}{N^{3}} \boldsymbol{\Gamma}[0, G]=\frac{1}{2} \ln G^{-1}+\frac{1}{2} G-\frac{1}{8} \lambda^{2} G^{4}+\frac{3 \lambda}{4 N^{1 / 2}} G^{2} .
$$




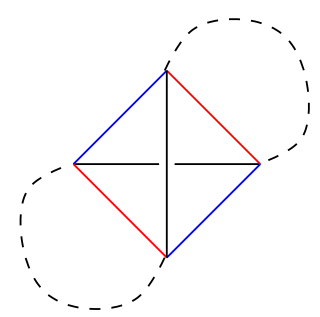

Figure 9. The NLO core graph for the CTKT model in the tetrahedron representation.

The equations of motion are now:

$$
G^{-1}=1-\lambda^{2} G^{3}+\frac{3 \lambda}{N^{1 / 2}} G .
$$

Writing $G=G^{(0)}+N^{-1 / 2} G^{(-1 / 2)}$ and expanding to order $N^{-1 / 2}$ we recover the SD equations at NLO of [59]. ${ }^{15}$

We expect to find an infinite family of graphs at NNLO, but the analysis of the CTKT model at NNLO has never been done and it goes beyond the scope of the present paper. Since, on the contrary, the subleading structure of the GW model is much better understood, we will study the subleading corrections (up to NNNLO) in that model in section 4.3.

\subsection{The fermionic CTKT model in $d=1$}

Let us consider the $d=1$ fermionic CTKT model, which is in some ways the simplest tensor model with SYK-like large- $N$ limit [22]. Its classical action is:

$$
\mathbf{S}_{\mathrm{CTKT}}[\psi]=\int_{t}\left(\frac{1}{2} \psi_{a b c}(t) \partial_{t} \psi_{a b c}(t)+\frac{\lambda}{4 N^{3 / 2}} \psi_{a_{1} a_{2} a_{3}}(t) \psi_{a_{1} b_{2} b_{3}}(t) \psi_{b_{1} a_{2} b_{3}}(t) \psi_{b_{1} b_{2} a_{3}}(t)\right)
$$

The selection of dominant graphs in the large- $N$ limit is not affected by the dimension of space time, hence the analysis of $d=0$ applies here without change. The Grassmann nature of the fields leads instead to some extra minus signs, just as in the SYK case.

We concentrate again on the symmetric phase $\underline{\Psi}=0$, which is the only possible one in $d=1$. At $\mathrm{LO}$ in the $1 / N$ expansion, $\boldsymbol{\Gamma}_{2}[0, G]$ is given again by a single diagram, the fundamental vacuum melon; with respect to (4.6) we only need to add the time dependence:

$$
\begin{aligned}
\Gamma_{2}^{(3)}[G] & =\frac{-\lambda^{2}}{8 N^{3}} \int_{t, t^{\prime}} G_{a_{1} a_{2} a_{3} b_{1} b_{2} b_{3}}\left(t, t^{\prime}\right) G_{a_{1} a_{2}^{\prime} a_{3}^{\prime} b_{1} b_{2}^{\prime} b_{3}^{\prime}}\left(t, t^{\prime}\right) G_{a_{1}^{\prime} a_{2} a_{3}^{\prime} b_{1}^{\prime} b_{2} b_{3}^{\prime}}\left(t, t^{\prime}\right) G_{a_{1}^{\prime} a_{2}^{\prime} a_{3} b_{1}^{\prime} b_{2}^{\prime} b_{3}}\left(t, t^{\prime}\right) \\
& =-\frac{1}{8} \lambda^{2} N^{3} \int_{t, t^{\prime}} G\left(t, t^{\prime}\right)^{4}
\end{aligned}
$$

where in the last equality we used a diagonal ansatz:

$$
G_{a_{1} a_{2} a_{3} b_{1} b_{2} b_{3}}\left(t, t^{\prime}\right)=G\left(t, t^{\prime}\right) \delta_{a_{1} b_{1}} \delta_{a_{2} b_{2}} \delta_{a_{3} b_{3}}
$$

\footnotetext{
${ }^{15} \mathrm{Up}$ to a factor of 3 which was forgotten in [59].
} 
which is valid on shell. By comparison with (3.8) it is obvious that we obtain the same behavior as in SYK, in particular the bilocal nature of the interaction. In fact, including also the one-loop contribution:

$$
\frac{1}{N^{3}} \boldsymbol{\Gamma}[0, G]=-\frac{1}{2} \operatorname{Tr}\left[\ln G^{-1}\right]-\frac{1}{2} \operatorname{Tr}\left[\partial_{t} G\left(t, t^{\prime}\right)\right]-\frac{1}{8} \lambda^{2} \int_{t, t^{\prime}} G\left(t, t^{\prime}\right)^{4},
$$

which has the same form as (3.10). If one were to not use a diagonal ansatz one would get:

$$
\boldsymbol{\Gamma}[0, G]=-\frac{1}{2} \operatorname{Tr}\left[\ln G_{a_{1} a_{2} a_{3} b_{1} b_{2} b_{3}}^{-1}\right]-\frac{1}{2} \operatorname{Tr}\left[\partial_{t} G_{a_{1} a_{2} a_{3} b_{1} b_{2} b_{3}}\left(t, t^{\prime}\right)\right]+\boldsymbol{\Gamma}_{2}^{(3)}[G] .
$$

with $\boldsymbol{\Gamma}_{2}^{(3)}[G]$ written as in the first line of (4.15).

As pointed out in [27], if in the infrared we discard the time-derivative term, the global $O(N)^{3}$ symmetry of (4.18) is promoted to a local symmetry. ${ }^{16}$ The would-be gauge degrees of freedom associated to such local transformations are however proper degrees of freedom due to the explicit breaking provided by the time-derivative term, which we expect to endow them with an effective action controlling their dynamics. The idea is very similar to what happens with conformal symmetry: the action (4.17) has precisely the same form as (3.10), and as such it is also conformally invariant (i.e. invariant under time reparametrizations) in the infrared/strong-coupling limit, i.e. when discarding the time-derivative term. The time derivative can then be viewed as a conformal breaking operator that generates an effective action for the conformal mode, which takes the form of a Schwarzian action $[3,5]$ (see $[9,13]$ for a derivation with an action with a single bilocal field, as in our (3.10), or [7] for more details on how to regularize the conformal breaking operator). Choudhury et al. [27] have followed a similar route to obtain an effective action for the would-be gauge degrees of freedom, arriving at a non-linear sigma model type action, as one would expect on general grounds. However, they postulated the action (4.18) as an effective classical action without any derivation, while we derived it here as a $2 \mathrm{PI}$ effective action. It is not clear at the moment whether a formulation analogous to the one in section 2.2 exists for the KTCT model, but we can see two limitations to it: first, we expect such a formulation to be necessarily more complicated in the tensor case, because there are many more invariants, and the large- $N$ expansion cannot be interpreted as a loop expansion; second, as we saw in $d=0$, the NLO correction to the 2PI effective action of the KTCT model is given by a finite number of graphs and therefore it does not have the form of the result of a one-loop integral (compare (4.12) with (2.41) or (3.18)), thus an hypothetical effective bilocal action would necessarily not factor the $N$-dependence as simply as in the vector case. However, we can bypass such open question, and apply the same reasoning directly to the $2 \mathrm{PI}$ effective action. In order to see why, it is useful to recall that in section 3 we found that $\boldsymbol{\Gamma}_{G G}$ gives the inverse 4-point function. The latter is then singular if $\boldsymbol{\Gamma}_{G G}$ has zero eigenvalues, as it is the case if there is a gauge invariance which has not been gauge-fixed. In the present case we do not need a gauge fixing because there is an explicit breaking of the gauge invariance. The would-be gauge modes give a non-zero contribution to the quadratic part of the action

\footnotetext{
${ }^{16}$ Notice that this does not happen in the SYK model: in eq. (3.8) the trace $G_{a a}\left(t, t^{\prime}\right)$ identifies indices at different times, while in (4.15) indices are identified at equal times.
} 
which can be obtained by evaluating the quadratic part of the breaking term in the gauge transformations around the stationary point.

In order to translate in formulas what we just said, we write:

$$
\begin{aligned}
\boldsymbol{\Gamma}_{\mathrm{inv}}[G] & =-\frac{1}{2} \operatorname{Tr}\left[\ln G_{a_{1} a_{2} a_{3} b_{1} b_{2} b_{3}}^{-1}\right]+\boldsymbol{\Gamma}_{2}^{(3)}[G], \\
\boldsymbol{\Gamma}_{\mathrm{pert}}[G] & =-\frac{1}{2} \operatorname{Tr}\left[\partial_{t} G_{a_{1} a_{2} a_{3} b_{1} b_{2} b_{3}}\left(t, t^{\prime}\right)\right]
\end{aligned}
$$

The stationary point of the total action splits as (using boldface for a collective index only for the tensor indices, e.g. $\left.\mathbf{a}=a_{1} a_{2} a_{3}\right)$ :

$$
\underline{G}_{\mathbf{a b}}=\underline{G}_{0}\left(t-t^{\prime}\right) \delta_{\mathbf{a b}}+\underline{G}_{1}\left(t-t^{\prime}\right) \delta_{\mathbf{a b}}
$$

where:

$$
\begin{aligned}
\frac{\delta \boldsymbol{\Gamma}_{\text {inv }}}{\delta G_{\mathbf{a b}}}\left[\underline{G}_{0}\right] & =0, \\
\frac{\delta^{2} \boldsymbol{\Gamma}_{\text {inv }}}{\delta G_{\mathbf{a b}} \delta G_{\mathbf{c c}}}\left[\underline{G}_{0}\right] \underline{G}_{1}+\frac{\delta \boldsymbol{\Gamma}_{\mathrm{pert}}}{\delta G_{\mathbf{a b}}}\left[\underline{G}_{0}\right] & =0 .
\end{aligned}
$$

We emphasize that $\underline{G}_{\mathbf{a b}}$ is leading order in $1 / N: \underline{G}_{1}$ is a perturbation in the strong coupling expansion, i.e. it arises by treating (4.20) as a perturbation to (4.19), but it is still leading order in the large $N$ limit. Next, consider the transformation:

$$
G_{\mathbf{a b}}\left(t, t^{\prime}\right) \rightarrow G_{\mathbf{a}^{\prime} \mathbf{b}^{\prime}}\left(t, t^{\prime}\right) \mathbb{V}_{\mathbf{a a}^{\prime}}(t) \mathbb{V}_{\mathbf{b b}^{\prime}}\left(t^{\prime}\right)
$$

where:

$$
\begin{aligned}
\mathbb{V}_{\mathbf{a c}}(t) & \equiv V_{a_{1} b_{1}}^{(1)}(t) V_{a_{2} b_{2}}^{(2)}(t) V_{a_{3} b_{3}}^{(3)}(t) \simeq \delta_{\mathbf{a b}}+\mathbb{H}_{\mathbf{a b}}(t)+\frac{1}{2} \mathbb{H}_{\mathbf{a c}}(t) \mathbb{H}_{\mathbf{c b}}(t)+\ldots \\
\mathbb{H}_{\mathbf{a b}}(t) & =H_{a_{1} b_{1}}^{(1)}(t) \delta_{a_{2} b_{2}} \delta_{a_{3} b_{3}}+\delta_{a_{1} b_{1}} H_{a_{2} b_{2}}^{(2)}(t) \delta_{a_{3} b_{3}}+\delta_{a_{1} b_{1}} \delta_{a_{2} b_{2}} H_{a_{3} b_{3}}^{(3)}(t)
\end{aligned}
$$

for $V_{a b}^{(i)} \in O(N)$ and $H_{a b}^{(i)}$ an antisymmetric matrix, for $i=1 \ldots 3$. Such transformation leaves $\boldsymbol{\Gamma}_{\text {inv }}[G]$ invariant, but not $\boldsymbol{\Gamma}_{\text {pert }}[G]$. Using the invariance of the former, and the linearity in $G$ of the latter, it can be easily shown (expanding at first order in $\underline{G}_{1}$ the left-hand-side and using (4.23)) that:

$$
\frac{\delta^{2}\left(\boldsymbol{\Gamma}_{\mathrm{inv}}+\boldsymbol{\Gamma}_{\mathrm{pert}}\right)}{\delta G_{\mathbf{a b}} \delta G_{\mathbf{c d}}}[\underline{G}] g_{\mathbf{a b}} g_{\mathbf{c d}}=\left.\frac{\delta^{2} \boldsymbol{\Gamma}_{\mathrm{pert}}\left[\underline{G}_{0} \mathbb{V}_{\mathbf{a c}} \mathbb{V}_{\mathbf{b c}}\right]}{\delta \mathbb{H}_{\mathbf{a b}} \delta \mathbb{H}_{\mathbf{c d}}}\right|_{\mathbb{H}=0} \mathbb{H}_{\mathbf{a b}} \mathbb{H}_{\mathbf{c d}}
$$

where:

$$
g_{\mathbf{a b}}=\underline{G}\left(t, t^{\prime}\right)\left(\mathbb{H}_{\mathbf{a b}}(t)-\mathbb{H}_{\mathbf{a b}}\left(t^{\prime}\right)\right) .
$$

Rewriting the quadratic part of $\underline{G}\left(t-t^{\prime}\right) \mathbb{V}_{\mathbf{a b}}(t) \mathbb{V}_{\mathbf{a b}}\left(t^{\prime}\right)$ as:

$$
\begin{aligned}
\frac{1}{2} \underline{G}\left(t-t^{\prime}\right)\left(\mathbb{H}_{\mathbf{a c}}(t) \mathbb{H}_{\mathbf{c a}}(t)+\mathbb{H}_{\mathbf{a c}}\left(t^{\prime}\right) \mathbb{H}_{\mathbf{c a}}\left(t^{\prime}\right)-2 \mathbb{H}_{\mathbf{a b}}(t) \mathbb{H}_{\mathbf{b a}}\left(t^{\prime}\right)\right) \\
\simeq \frac{1}{2} \underline{G}\left(t-t^{\prime}\right)\left(\partial_{t} \mathbb{H}_{\mathbf{a c}}(t) \partial_{t} \mathbb{H}_{\mathbf{c a}}(t)\left(t-t^{\prime}\right)^{2}+O\left(\left(t-t^{\prime}\right)^{3}\right)\right)
\end{aligned}
$$



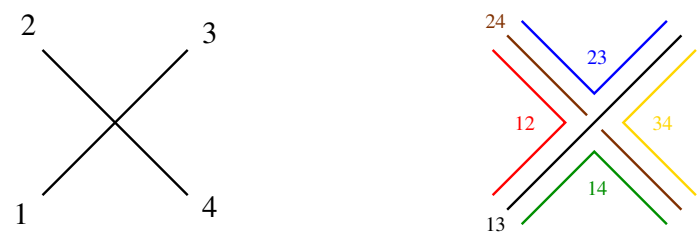

Figure 10. The vertex of the GW model (4.32) with $q=4$ in the Feynman (left) and the stranded (right) representations.

we obtain:

$$
\left.\frac{\delta^{2} \boldsymbol{\Gamma}_{\mathrm{pert}}\left[\underline{G}_{0} \mathbb{V}_{\mathbf{a c}} \mathbb{V}_{\mathbf{b c}}\right]}{\delta \mathbb{H}_{\mathbf{a b}} \delta \mathbb{H}_{\mathbf{c d}}}\right|_{\mathbb{H}=0} \mathbb{H}_{\mathbf{a b}} \mathbb{H}_{\mathbf{c d}}=-\frac{\alpha}{2} \int_{t} \partial_{t} \mathbb{H}_{\mathbf{a c}}(t) \partial_{t} \mathbb{H}_{\mathbf{c a}}(t)
$$

where

$$
\alpha=\int_{\tau} \underline{G}_{0}(\tau) \tau^{2} \sigma(\tau),
$$

with $\sigma(\tau)$ a suitable regularization of $\delta^{\prime}(\tau)$. This is precisely the same coefficient that appears in front of the Schwarzian action, as derived in [7], and the action coincides with the one derived in [27].

\subsection{The fermionic GW model in $d=1$}

The (real) GW model in one dimension is defined by the action:

$$
\mathbf{S}_{\mathrm{GW}}[\psi]=\frac{1}{2} \sum_{c=1}^{q} \int_{t, t^{\prime}} \psi_{\mathbf{a}_{\mathbf{c}}}^{(c)}(t) C^{-1}\left(t, t^{\prime}\right) \psi_{\mathbf{a}_{\mathbf{c}}}^{(c)}\left(t^{\prime}\right)+\frac{\mathrm{i}^{q / 2} \lambda}{N^{(q-1)(q-2) / 4}} \int_{t} \prod_{c=1}^{q} \psi_{\mathbf{a}_{\mathbf{c}}}^{(c)}(t) \prod_{c_{1}<c_{2}} \delta_{a_{c_{1} c_{2}} a_{c_{2} c_{1}}},
$$

where $\mathbf{a}_{\mathbf{c}}=\left(a_{c c_{1}} \mid c_{1} \in\{1, \ldots, q\} \backslash\{c\}\right)$ and $C^{-1}\left(t, t^{\prime}\right)=\partial_{t} \delta\left(t-t^{\prime}\right)$. The vertex is represented in figure 10 for the case $q=4$. The model is symmetric under the global group $O(N)^{q(q-1) / 2}$, where an independent $O(N)$ element acts on each pair $\left(a_{c_{1} c_{2}}, a_{c_{2} c_{1}}\right)$.

We introduce a bilocal source for each color and obtain the 2PI effective action:

$$
\boldsymbol{\Gamma}\left[\Psi^{(c)}, G^{(c)}\right]=\mathbf{S}_{\mathrm{GW}}\left[\Psi^{(c)}\right]+\frac{1}{2} \sum_{c=1}^{q} \operatorname{Tr}\left[\ln \left(G^{(c)}\right)\right]-\frac{1}{2} \sum_{c=1}^{q} \operatorname{Tr}\left[\left(G_{0}^{(c)}\right)^{-1} G^{(c)}\right]+\boldsymbol{\Gamma}_{2}\left[\Psi^{(c)}, G^{(c)}\right] .
$$

From now on we consider the symmetric phase $\underline{\Psi}^{(c)}=0$. The leading order 2PI graph is again the fundamental melon, thus $\Gamma_{2}\left[0, G^{(c)}\right]$ has a large- $N$ expansion which starts at order $N^{q-1}$ :

$$
\begin{aligned}
\Gamma_{2}^{(q-1)}\left[0, G^{(c)}\right] & =-\frac{\lambda^{2}}{2 N^{(q-1)(q-2) / 2}} \int_{t, t^{\prime}} \prod_{c=1}^{q} G_{\mathbf{a}_{c} \mathbf{b}_{\mathbf{c}}}^{(c)}\left(t, t^{\prime}\right) \prod_{c_{1}<c_{2}} \delta_{a_{c_{1} c_{2}} a_{c_{2} c_{1}}} \delta_{b_{c_{1} c_{2}} b_{c_{2} c_{1}}} \\
& =-\frac{\lambda^{2} N^{q-1}}{2} \int_{t, t^{\prime}} G\left(t, t^{\prime}\right)^{q}
\end{aligned}
$$

where in the last step we restricted to the color symmetric and diagonal ansatz $G_{\mathbf{a}_{\mathbf{c}} \mathbf{b}_{\mathbf{c}}}^{(c)}\left(t, t^{\prime}\right)=$ $G\left(t, t^{\prime}\right) \prod_{c^{\prime} \neq c} \delta_{a_{c c^{\prime}} b_{c c^{\prime}}}$. With such ansatz we recover precisely the SYK result (3.10), with $J$ replaced by $\lambda$ and $N$ by $q N^{q-1}$. 
As $-\boldsymbol{\Gamma}_{2}$ is the sum over $2 \mathrm{PI}$ vacuum graphs, the subleading corrections begin at order $N^{2}$ for any $q$ [40], and the diagrams contributing to $\boldsymbol{\Gamma}_{2}^{(2)}, \boldsymbol{\Gamma}_{2}^{(1)}$ and $\boldsymbol{\Gamma}_{2}^{(0)}$ are all the ring graphs consisting in 4-point ladder diagrams closing onto themselves, similar to the ones depicted in figure 6 , but in which we need to distinguish the various possible sequences of colors along the rails [40]. In order to compute their contributions to the 2PI effective action, we recall that the Gaussian expectation with covariance $G$ of real fermions is:

$$
\left\langle\psi\left(t_{1}\right) \ldots \psi\left(t_{2 n}\right)\right\rangle=\sum_{\pi} \epsilon(\pi) \prod_{(k, l) \in \pi} G\left(t_{k}, t_{l}\right)
$$

where $\pi$ are the pairings of $2 n$ elements, $(k, l)$ with $k<l$ and $\epsilon(\pi)$ is the signature of the pairing. We are interested in the perturbative expansion at order $2 n$ :

$$
\frac{\lambda^{2 n} \mathrm{i}^{n q}}{(2 n) !}\left\langle\prod_{i=1}^{2 n} \psi^{1}\left(t_{i}\right) \ldots \psi^{q}\left(t_{i}\right)\right\rangle_{\text {ring }},
$$

where the subscript signals that we only select the contractions that reproduce ring graphs.

A ring graph is built by first pairing the $2 n$ vertices into $n$ pairs where the vertices in a pair are connected by $q-2$ edges. We denote the colors of the external edges of a dipole $c_{1}$ and $c_{2}$. For each pair we get a factor:

$$
\begin{aligned}
(-1)^{q / 2+q(q-1) / 2} \psi^{c_{1}}(t) \psi^{c_{2}}(t) & {\left[G\left(t, t^{\prime}\right)\right]^{q-2} \psi^{c_{2}}\left(t^{\prime}\right) \psi^{c_{1}}\left(t^{\prime}\right) } \\
& =\psi^{c_{1}}(t) \psi^{c_{1}}\left(t^{\prime}\right)(-1)\left[G\left(t, t^{\prime}\right)\right]^{q-2} \psi^{c_{2}}\left(t^{\prime}\right) \psi^{c_{2}}(t) .
\end{aligned}
$$

We now glue the pairs together to form ring graphs. This identifies the right external colors on a pair with the left external colors on the next pair. The field at $t_{i}$ can connect with either $t_{i+1}$ or $t_{i+1}^{\prime}$ and we obtain schematically:

$$
\begin{aligned}
\frac{\lambda^{2 n}}{(2 n) !} \frac{(2 n) !}{2^{n} n !}(n-1) ! 2^{n-1} \sum_{\left\{c_{i}\right\}}^{c_{i} \neq c_{i-1}}( & \left.\prod_{i=1}^{n} G\left(t_{i-1}, t_{i}\right) G\left(t_{i-1}^{\prime}, t_{i}^{\prime}\right)(-1)\left[G\left(t_{i}, t_{i}^{\prime}\right)\right]^{q-2}\right) \\
& \times\left[\delta\left(t_{n}-t_{0}\right) \delta\left(t_{n}^{\prime}-t_{0}^{\prime}\right)-\delta\left(t_{n}-t_{0}^{\prime}\right) \delta\left(t_{n}^{\prime}-t_{0}\right)\right] .
\end{aligned}
$$

We now reinstate the tensor indices. We denote:

$$
\begin{aligned}
& \hat{\mathbb{K}}_{\mathbf{a}_{\mathbf{c}_{\mathbf{1}}} \mathbf{a}_{\mathbf{c}_{\mathbf{1}}}^{\prime} ; \mathbf{b}_{\mathbf{c}_{\mathbf{2}}} \mathbf{b}_{\mathbf{c}_{\mathbf{2}}}^{\prime}}^{\left(c_{1} c_{2}\right.}\left(t_{a}, t_{a^{\prime}} ; t_{b}, t_{b^{\prime}}\right)=(-1) G_{\mathbf{a}_{\mathbf{c}_{\mathbf{1}}} \mathbf{b}_{\mathbf{c}_{\mathbf{1}}}}^{(c)}\left(t_{a}, t_{b}\right) G_{\mathbf{a}_{\mathbf{c}_{\mathbf{1}}}^{\prime} \mathbf{b}_{\mathbf{c}_{\mathbf{1}}}^{\prime}}^{(c)}\left(t_{a^{\prime}}, t_{b^{\prime}}\right) \\
& \times\left(\prod_{c \neq c_{1}, c_{2}} G_{\mathbf{b}_{\mathbf{c}} \mathbf{b}_{\mathbf{c}}^{\prime}}^{(c)}\left(t_{b}, t_{b^{\prime}}\right)\right)\left(\prod_{c<c^{\prime}} \delta_{b_{c c^{\prime}} b_{c^{\prime} c}} \delta_{b_{c c^{\prime}}{ }^{\prime} b_{c^{\prime} c}^{\prime}}\right), \\
& \underset{\mathbf{a}_{\mathbf{a}_{\mathbf{a}} \mathbf{a}_{\mathbf{c}}^{\prime} ; \mathbf{b}_{\mathbf{c}} \mathbf{b}_{\mathbf{c}}^{\prime}}^{=}}{=}\left(t_{a}, t_{a^{\prime}} ; t_{b}, t_{b^{\prime}}\right)=\delta_{\mathbf{a}_{\mathbf{c}} \mathbf{b}_{\mathbf{c}}} \delta_{\mathbf{a}_{\mathbf{c}}^{\prime} \mathbf{b}_{\mathbf{c}}^{\prime}} \delta\left(t_{a}-t_{b}\right) \delta\left(t_{a^{\prime}}-t_{b^{\prime}}\right), \\
& \underset{\mathbf{a}_{\mathbf{c}} \mathbf{a}_{\mathbf{c}}^{\prime} ; \mathbf{b}_{\mathbf{c}} \mathbf{b}_{\mathbf{c}}^{\prime}}{\mathbb{1}}\left(t_{a}, t_{a^{\prime}} ; t_{b}, t_{b^{\prime}}\right)=\delta_{\mathbf{a}_{\mathbf{c}} \mathbf{b}_{\mathbf{c}}^{\prime}} \delta_{\mathbf{a}_{\mathbf{c}}^{\prime} \mathbf{b}_{\mathbf{c}}} \delta\left(t_{a}-t_{b^{\prime}}\right) \delta\left(t_{a^{\prime}}-t_{b}\right),
\end{aligned}
$$


where repeated indices are summed. Later on we will take the color symmetric diagonal ansatz for the two point function. We denote:

$$
\begin{aligned}
\hat{\mathcal{K}}\left(t_{a}, t_{a^{\prime}} ; t_{b}, t_{b^{\prime}}\right) & =(-1) G\left(t_{a}, t_{b}\right) G\left(t_{a^{\prime}}, t_{b^{\prime}}\right)\left[G\left(t_{b}, t_{b^{\prime}}\right)\right]^{q-2}, \\
I^{=}\left(t_{a}, t_{a^{\prime}} ; t_{b}, t_{b^{\prime}}\right) & =\delta\left(t_{a}-t_{b}\right) \delta\left(t_{a^{\prime}}-t_{b^{\prime}}\right), \\
I^{\times}\left(t_{a}, t_{a^{\prime}} ; t_{b}, t_{b^{\prime}}\right) & =\delta\left(t_{a}-t_{b^{\prime}}\right) \delta\left(t_{a^{\prime}}-t_{b}\right),
\end{aligned}
$$

and $\hat{\mathcal{K}} I^{\times}=I^{\times} \hat{\mathcal{K}}$. As a function of the sequence of horizontal colors $c_{i}$, as well as the last contraction, we get the following contributions to the $2 \mathrm{PI}$ effective action:

$$
\begin{aligned}
& \Gamma_{2}^{(2)}=-\sum_{n \geq 2} \frac{\lambda^{2 n}}{2 n} N^{-n(q-1)(q-2) / 2} \sum_{\left(c_{1} \ldots c_{n}\right) \in U_{n}}^{c_{n+1}=c_{1}} \operatorname{Tr}\left[\left(\prod_{i=1}^{n} \hat{\mathbb{K}}^{\left(c_{i} c_{i+1}\right)}\right) \mathbb{I}^{=}\right], \\
& \boldsymbol{\Gamma}_{2}^{(1)}=-\sum_{n \geq 2} \frac{\lambda^{2 n}}{2 n} N^{-n(q-1)(q-2) / 2} \sum_{\left(c_{n} \ldots c_{n}\right) \in U_{n}}^{c_{n+1}=c_{1}} \operatorname{Tr}\left[\left(\prod_{i=1}^{n} \hat{\mathbb{K}}^{\left(c_{i} c_{i+1}\right)}\right)\left(-\mathbb{I}^{\times}\right)\right], \\
& \boldsymbol{\Gamma}_{2}^{(0)}=-\sum_{n \geq 2} \frac{\lambda^{2 n}}{2 n} N^{-n(q-1)(q-2) / 2} \sum_{\left(c_{1} \ldots c_{n}\right) \in B_{n}}^{c_{n+1}=c_{1}} \operatorname{Tr}\left[\left(\prod_{i=1}^{n} \hat{\mathbb{K}}^{\left(c_{i} c_{i+1}\right)}\right)\left(\mathbb{I}^{=}-\mathbb{I}^{\times}\right)\right],
\end{aligned}
$$

where $U_{n}$ is the set of alternating (or unbroken) words $\left(c_{1} c_{2} \ldots c_{1} c_{2}\right)$ of length $n$ with $c_{1}<c_{2}$ over the colors, and $B_{n}$ is the set of non alternating (or broken) words $\left(c_{1} \ldots c_{3} \ldots c_{2}\right), \quad c_{i} \neq$ $c_{i+1}$ of length $n$ with $c_{1}<c_{2}$ over the colors. Restricting to the color symmetric diagonal ansatz we get:

$$
\begin{aligned}
& \boldsymbol{\Gamma}_{2}^{(2)}=-N^{2} \sum_{n \geq 2} \frac{\lambda^{2 n}}{2 n}\left|U_{n}\right| \operatorname{Tr}\left[\hat{\mathcal{K}}^{n} I^{=}\right], \\
& \boldsymbol{\Gamma}_{2}^{(1)}=-N \sum_{n \geq 2} \frac{\lambda^{2 n}}{2 n}\left|U_{n}\right| \operatorname{Tr}\left[\hat{\mathcal{K}}^{n}\left(-I^{\times}\right)\right], \\
& \boldsymbol{\Gamma}_{2}^{(0)}=-\sum_{n \geq 2} \frac{\lambda^{2 n}}{2 n}\left|B_{n}\right| \operatorname{Tr}\left[\hat{\mathcal{K}}^{n}\left(I^{=}-I^{\times}\right)\right] .
\end{aligned}
$$

In all these cases $-N^{-r} \lambda \partial_{\lambda} \boldsymbol{\Gamma}_{2}^{(r)}$ is a generating function of nonempty words with weight $\lambda^{2} \hat{\mathcal{K}}$ per letter. Ignoring for an instant the fact that $\hat{\mathcal{K}}$ is an operator and denoting in superscript the two external letters of the word we have:

- Unbroken words. The generating functions of nonempty, unbroken words are simple geometric series:

$$
U^{c_{1} c_{1}}=\frac{(q-1) \lambda^{6} \hat{\mathcal{K}}^{3}}{1-\lambda^{4} \hat{\mathcal{K}}^{2}}, \quad U^{c_{1} c_{2}}=\frac{\lambda^{4} \hat{\mathcal{K}}^{2}}{1-\lambda^{4} \hat{\mathcal{K}}^{2}}=\lambda \partial_{\lambda}\left[-\frac{1}{4} \ln \left(1-\lambda^{4} \hat{\mathcal{K}}^{2}\right)\right]
$$

- Arbitrary words. The generating function of nonempty, arbitrary words with equal external letters is:

$$
A^{c_{1} c_{1}}=\lambda^{2} \hat{\mathcal{K}} \frac{(q-1) \lambda^{2} \hat{\mathcal{K}}}{1-(q-2) \lambda^{2} \hat{\mathcal{K}}}\left(\lambda^{2} \hat{\mathcal{K}}+A^{c_{1} c_{1}}\right)=\frac{(q-1) \lambda^{6} \hat{\mathcal{K}}^{3}}{1-(q-2) \lambda^{2} \hat{\mathcal{K}}-(q-1) \lambda^{4} \hat{\mathcal{K}}^{2}},
$$


because an arbitrary, non empty word with equal external letters $c_{1}$ is: a letter $c_{1}$ followed by a nonempty word which does not reuse the letter $c_{1}$, followed by either exactly a letter $c_{1}$ or a nonempty word with external letters $c_{1} c_{1}$. The generating function of nonempty, arbitrary words with different external letters is:

$$
\begin{aligned}
A^{c_{1} c_{2}} & =\lambda^{2} \hat{\mathcal{K}}\left[\frac{1}{1-(q-2) \lambda^{2} \hat{\mathcal{K}}}\right]\left(\lambda^{2} \hat{\mathcal{K}}+A^{c_{2} c_{2}}\right)=\frac{\lambda^{4} \hat{\mathcal{K}}^{2}}{1-(q-2) \lambda^{2} \hat{\mathcal{K}}-(q-1) \lambda^{4} \hat{\mathcal{K}}^{2}} \\
& =\lambda \partial_{\lambda}\left[-\frac{1}{2 q(q-1)} \ln \left[1-(q-1) \lambda^{2} \hat{\mathcal{K}}\right]-\frac{1}{2 q} \ln \left(1+\lambda^{2} \hat{\mathcal{K}}\right)\right]
\end{aligned}
$$

as such a word is a letter $c_{1}$ followed by a possibly empty word which does not use the letter $c_{2}$, followed by either a letter $c_{2}$ or a nonempty word with external letters $c_{2} c_{2}$.

- Broken words. The generating function of nonempty, broken words with different external letters is:

$$
\begin{aligned}
B^{c_{1} c_{2}} & =A^{c_{1} c_{2}}-U^{c_{1} c_{2}} \\
& =\lambda \partial_{\lambda}\left[-\frac{1}{2 q(q-1)} \ln \left[1-(q-1) \lambda^{2} \hat{\mathcal{K}}\right]-\frac{1}{2 q} \ln \left(1+\lambda^{2} \hat{\mathcal{K}}\right)+\frac{1}{4} \ln \left(1-\lambda^{4} \hat{\mathcal{K}}^{2}\right)\right] .
\end{aligned}
$$

Recalling now that $\hat{\mathcal{K}}$ is an operator and that the projector on antisymmetric functions is $I_{-}=\left(I^{=}-I^{\times}\right) / 2$ we get with the color-symmetric diagonal ansatz:

$$
\boldsymbol{\Gamma}[0, G]=N^{q-1} \frac{q}{2} \operatorname{Tr}[\ln (G)]-N^{q-1} \frac{q}{2} \operatorname{Tr}\left[\partial_{t} G\right]+\boldsymbol{\Gamma}_{2}[G],
$$

where:

$$
\boldsymbol{\Gamma}_{2}[G]=\boldsymbol{\Gamma}_{2}^{(q-1)}[G]+\boldsymbol{\Gamma}_{2}^{(2)}[G]+\boldsymbol{\Gamma}_{2}^{(1)}[G]+\boldsymbol{\Gamma}_{2}^{(0)}[G],
$$

and:

$$
\begin{aligned}
\Gamma_{2}^{(q-1)}[G]= & N^{q-1}\left(-\frac{\lambda^{2}}{2}\right) \int_{t, t^{\prime}} G\left(t, t^{\prime}\right)^{q}, \\
\Gamma_{2}^{(2)}[G]= & N^{2} \frac{1}{4}\left(\begin{array}{l}
q \\
2
\end{array}\right) \operatorname{Tr}\left[I^{=} \ln \left(1-\lambda^{4} \hat{\mathcal{K}}^{2}\right)\right], \\
\boldsymbol{\Gamma}_{2}^{(1)}[G]= & N \frac{1}{4}\left(\begin{array}{l}
q \\
2
\end{array}\right) \operatorname{Tr}\left[\left(-I^{\times}\right) \ln \left(1-\lambda^{4} \hat{\mathcal{K}}^{2}\right)\right], \\
\boldsymbol{\Gamma}_{2}^{(0)}[G]= & \frac{1}{2} \operatorname{Tr}\left[I_{-} \ln \left(1-(q-1) \lambda^{2} \hat{\mathcal{K}}\right)\right], \\
& +\frac{q-1}{2} \operatorname{Tr}\left[I_{-} \ln \left(1+\lambda^{2} \hat{\mathcal{K}}\right)\right]-\frac{1}{2}\left(\begin{array}{l}
q \\
2
\end{array}\right) \operatorname{Tr}\left[I_{-} \ln \left(1-\lambda^{4} \hat{\mathcal{K}}^{2}\right)\right] .
\end{aligned}
$$

A first use of eq. (4.48) is to determine the two point function at subleading order in $1 / N$. For instance, truncating the equation $\partial_{G} \boldsymbol{\Gamma}=0$ at leading and next to leading order for the bosonic GW model in $d=0$ by we obtain:

$$
0=-G^{-1}+1-\lambda^{2} G^{q-1}-\frac{1}{N^{q-3}}\left(\begin{array}{l}
q \\
2
\end{array}\right) \frac{\lambda^{4} G^{2 q-1}}{1-\lambda^{4} G^{2 q}},
$$


and substituting $G=\underline{G}^{(0)}+N^{-q+3} \underline{G}^{(-q+3)}$, with $\underline{G}^{(0)}=1+\lambda^{2}\left(\underline{G}^{(0)}\right)^{q}$, we get:

$$
\underline{G}^{(-q+3)}=\frac{1}{N^{q-3}}\left(\begin{array}{l}
q \\
2
\end{array}\right) \frac{\lambda^{4}\left(\underline{G}^{(0)}\right)^{2 q}}{\left[1-\lambda^{4}\left(\underline{G}^{(0)}\right)^{2 q}\right]\left[1-q \lambda^{2}\left(\underline{G}^{(0)}\right)^{q-1}\right]},
$$

reproducing the result of [66].

Going back to $d=1$ we observe that $\left.\partial_{G} \boldsymbol{\Gamma}^{(q-1)}\right|_{G=G^{(0)}}=0$, hence $\boldsymbol{\Gamma}\left[0, \underline{G}^{(0)}+\right.$ $\left.N^{-q+3} \underline{G}^{(-q+3)}\right]=\boldsymbol{\Gamma}\left[0, \underline{G}^{(0)}\right]$ up to terms of order $N^{q-1}\left(N^{-q+3} \underline{G}^{(-q+3)}\right)^{2} \sim N^{5-q}\left(\underline{G}^{(-q+3)}\right)^{2}$, that is (except for $q=4$ which is special) subleading with respect to all the terms in eq. (4.48). Thus, up to order $N^{0}$, the free energy of the GW model with $q \geq 6$ is (introducing also the projector on symmetric functions $\left.I_{+}=\left(I^{=}+I^{\times}\right) / 2\right)$ :

$$
\begin{aligned}
-\ln Z= & N^{q-1} \frac{q}{2} \operatorname{Tr}\left[\ln \left(\underline{G}^{(0)}\right)\right]-N^{q-1} \frac{q}{2} \operatorname{Tr}\left[\partial_{t} \underline{G}^{(0)}\right]-N^{q-1} \frac{\lambda^{2}}{2} \int_{t, t^{\prime}} \underline{G}^{(0)}\left(t, t^{\prime}\right)^{q} \\
& +\left[\frac{N(N-1)}{2}\left(\begin{array}{l}
q \\
2
\end{array}\right)\right] \frac{1}{2} \operatorname{Tr}\left[\ln \left(1-\lambda^{4}\left[\underline{\hat{\mathcal{K}}}^{(0)}\right]^{2} I_{+}\right)\right] \\
& +\left[\left(\frac{N(N-1)}{2}+(N-1)\right)\left(\begin{array}{l}
q \\
2
\end{array}\right)\right] \frac{1}{2} \operatorname{Tr}\left[\ln \left(1-\lambda^{4}\left[\underline{\hat{\mathcal{K}}}^{(0)}\right]^{2} I_{-}\right)\right] \\
& +(q-1) \frac{1}{2} \operatorname{Tr}\left[\ln \left(1+\lambda^{2}\left[\underline{\hat{\mathcal{K}}}^{(0)}\right] I_{-}\right)\right]+\frac{1}{2} \operatorname{Tr}\left[\ln \left(1-(q-1) \lambda^{2}\left[\underline{\hat{\mathcal{K}}}^{(0)}\right] I_{-}\right)\right],
\end{aligned}
$$

where the four point kernel $\underline{\hat{\mathcal{K}}}^{(0)}$ is evaluated on $\underline{G}^{(0)}$, the on-shell leading-order two point function, and where we have rearranged the subleading terms in order to eliminate $I^{=}$and $I^{\times}$in favor of $I_{ \pm}$. For $q=4$ the terms of order $N$ and order 1 receive corrections from $\underline{G}^{(-1)}$. All the subleading correction have the form of traces of a logarithm, hence each of them can be interpreted as resulting from the integration of freely fluctuating bilocal fields. Furthermore, the factor $\frac{N(N-1)}{2}\left(\begin{array}{l}q \\ 2\end{array}\right)$ in the second line is very suggestive of the number of antisymmetric matrices on color $i j, i \neq j$, while the factor $\left(\frac{N(N-1)}{2}+(N-1)\right)\left(\begin{array}{l}q \\ 2\end{array}\right)$ in the third line is suggestive of the number of symmetric traceless matrices on the same colors. Such interpretation is in fact correct, as we will now show.

It turns out that the final result (4.51) can be interpreted as a one-loop approximation for a bilocal effective action of the same form as the $2 \mathrm{PI}$ effective action at LO:

$$
\begin{aligned}
\mathbf{S}_{\mathrm{eff}}[G]= & \frac{1}{2} \sum_{c=1}^{q} \operatorname{Tr}\left[\ln \left(G^{(c)}\right)\right]-\frac{1}{2} \sum_{c=1}^{q} \operatorname{Tr}\left[\left(G_{0}^{(c)}\right)^{-1} G^{(c)}\right] \\
& -\frac{\lambda^{2}}{2 N^{(q-1)(q-2) / 2}} \int_{t, t^{\prime}} \prod_{c=1}^{q} G_{\mathbf{a}_{\mathbf{c}} \mathbf{b}_{\mathbf{c}}}^{(c)}\left(t, t^{\prime}\right) \prod_{c_{1}<c_{2}} \delta_{a_{c_{1} c_{2}} a_{c_{2} c_{1}}} \delta_{b_{c_{1} c_{2}} b_{c_{2} c_{1}}} .
\end{aligned}
$$

In order to see that, we split the bilocal field as on-shell background plus fluctuations,

$$
G_{\mathbf{a}_{\mathbf{c}} \mathbf{b}_{\mathbf{c}}}^{(c)}\left(t, t^{\prime}\right)=\underline{G}^{(0)}\left(t, t^{\prime}\right) \delta_{\mathbf{a}_{\mathbf{c}} \mathbf{b}_{\mathbf{c}}}+g_{\mathbf{a}_{\mathbf{c}} \mathbf{b}_{\mathbf{c}}}^{(c)}\left(t, t^{\prime}\right),
$$

and expand the action to second order in the fluctuations $g_{\mathbf{a}_{\mathbf{c}} \mathbf{b}_{\mathbf{c}}}^{(c)}\left(t, t^{\prime}\right)$. We obtain a quadratic action of the form (see appendix A for notation):

$$
\left\langle\mathbf{g}\left|\mathbb{B}\left(\mathbb{I}-\lambda^{2} \mathbb{K}\right)\right| \mathbf{g}\right\rangle,
$$




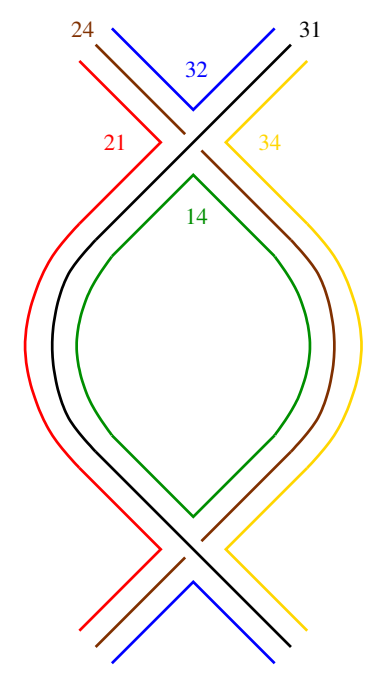

Figure 11. The kernel $\mathcal{K}^{\left(c_{1} c_{2}\right)}$ for $q=4$ and $\left(c_{1} c_{2}\right)=(23)$. When "gluing" to its right a fluctuation

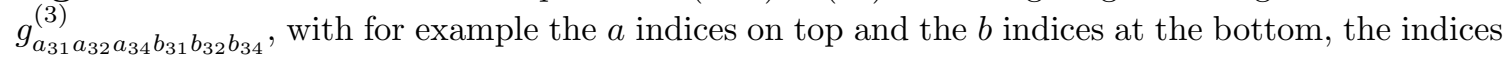
of color 31 and 34 are traced, while the index of color 32 is transmitted.

where $\mathbb{B}$ is a $\lambda$-independent $q \times q$ block matrix with $\left(\underline{G}^{(0)}\right)^{-1}\left(\underline{G}^{(0)}\right)^{-1}$ on its diagonal and zero otherwise. The latter leads to a $\frac{1}{2} \operatorname{Tr} \ln \mathbb{B}$ term in the free energy that should be canceled by the measure, as for zero coupling $\boldsymbol{\Gamma}_{2}[G]$ should vanish. Notice that in the case of the $O(N)$ and SYK models we obtained the correct measure thanks to the Lagrange multiplier $\tilde{\Sigma}$; we could introduce a similar field here by analogy, but since we are not deriving $\mathbf{S}_{\text {eff }}$ directly from the path integral it seems more natural to just fix the normalization by the zero-coupling condition. Therefore, we can replace $\mathbb{B}=1$ in (4.54).

The important point to notice is that the operator $\mathbb{K}$ in (4.54) is built out of kernels $\mathcal{K}^{\left(c_{1} c_{2}\right)}$ that when acting on $g_{\mathbf{a}_{\mathbf{c}} \mathbf{b}_{\mathbf{c}}}^{\left(c_{1}\right)}\left(t, t^{\prime}\right)$ or $g_{\mathbf{a}_{\mathbf{c}} \mathbf{b}_{\mathbf{c}}}^{\left(c^{2}\right)}\left(t, t^{\prime}\right)$ take their trace with respect to all the indices of color different from $c_{1} c_{2}$ (see figure 11). Therefore, it is useful to decompose (see appendix A for details):

$$
g_{\mathbf{a}_{\mathbf{c}} \mathbf{b}_{\mathbf{c}}}^{(c)}\left(t, t^{\prime}\right)=g^{(c)}\left(t, t^{\prime}\right) \prod_{i \neq c} \delta_{a_{c i} b_{c i}}+\sum_{i \neq c} g_{a_{c i} b_{c i}}^{(c i)}\left(t, t^{\prime}\right) \prod_{j \neq i, c} \delta_{a_{c j} b_{c j}}+\hat{g}_{\mathbf{a}_{\mathbf{c}} \mathbf{b}_{\mathbf{c}}}^{(c)}\left(t, t^{\prime}\right)
$$

where $g_{a_{c i} a_{c i}}^{(c i)}\left(t, t^{\prime}\right)=0$, for any $i$, and $\hat{g}_{\mathbf{a}_{\mathbf{c}} \mathbf{b}_{\mathbf{c}}}^{(c)}\left(t, t^{\prime}\right) \prod_{j \neq i} \delta_{a_{c j} b_{c j}}=0$, for any $i \neq c$.

One can then further decompose $g_{a_{c i} b_{c i}}^{(c i)}\left(t, t^{\prime}\right)$ in symmetric traceless and antisymmetric parts with respect to the matrix indices (since $g_{a_{c i} b_{c i}}^{(c i)}\left(t, t^{\prime}\right)=-g_{b_{c i} a_{c i}}^{(c i)}\left(t^{\prime}, t\right)$, the symmetry properties with respect to $t$ and $t^{\prime}$ are opposite to those of the indices).

The Hessian has a block-diagonal form corresponding to the decomposition (4.55). The block corresponding to the scalar modes $g^{(c)}\left(t, t^{\prime}\right)$ is a $q \times q$ matrix with the identity operator $I_{-}$on the diagonal entries, and $-\lambda^{2} \underline{\hat{\mathcal{K}}}^{(0)} I_{-}$on the off-diagonal ones. Such a matrix has one eigenvalue $\left(1-(q-1) \lambda^{2} \underline{\hat{\mathcal{K}}}^{(0)}\right) I_{-}$, and $(q-1)$ eigenvalues $\left(1+\lambda^{2} \underline{\hat{\mathcal{K}}}^{(0)}\right) I_{-}$, thus upon integration of such modes we obtain the last line in (4.51). The blocks corresponding to the matrix modes $g_{a_{c i} b_{c i}}^{\left(c_{1} c_{2}\right)}\left(t, t^{\prime}\right)$, are instead $2 \times 2$ matrices for each fixed pair $c_{1} c_{2}$, with the identity operator $\Im \equiv \mathcal{S} I_{-}+\mathcal{A} I_{+}$(the operators $\mathcal{S}$ and $\mathcal{A}$ are the projectors on symmetric 
traceless and antisymmetric matrices, respectively) on the diagonal entries, and $-\lambda^{2} \underline{\hat{\mathcal{K}}}^{(0)} \Im$ on the off-diagonal ones. Integration over such modes thus produces the second and third line of (4.51). Lastly, the block corresponding to $\hat{g}_{\mathbf{a}_{\mathbf{c}} \mathbf{b}_{\mathbf{c}}}^{(c)}\left(t, t^{\prime}\right)$ is just the identity, hence it does not lead to any subleading correction to the free energy.

\section{Summary and outlook}

We have introduced and discussed the 2PI effective action for the SYK model and for tensor field theories. The main lessons we drew from that are:

- For the SYK model, the 2PI effective action easily reproduces all the results of the bilocal action formalism [5, 7, 9], without using the replica method, at least up to the same order in $1 / N$ at which the replica symmetric ansatz works for the latter.

- For tensor analogues of the SYK model, the 2PI formalism offers so far the only way to obtain an effective action for collective fields, and it allows to obtain the same type of results as in the SYK model.

- For the CTKT model, the 2PI effective action provides a solid starting point for the argument of [27], showing the existence of soft modes associated to the $O(N)^{3}$ quasigauge invariance in the strong coupling limit. A similar argument can be repeated straightforwardly for the GW model with the symmetry group being replaced by $O(N)^{q(q-1) / 2}$.

- For the GW model, the $1 / N$ expansion of the 2PI effective action can be pushed up to NNNLO, and for all three subleading orders we find traces of logarithms, which have a natural interpretation as the result of Gaussian integrals over bilocal fields. Somewhat surprisingly, such Gaussian integrals correspond precisely to the one-loop approximation for a bilocal effective action of the same form as the leading-order 2PI effective action.

We think that the 2PI formalism is particularly promising for the exploration of subleading effects in $1 / N$ in tensor field theories. Hopefully this can lead to a better understanding of the underlying degrees of freedom and their possible holographic interpretation. It would also be interesting to carry out a NNLO analysis for the CTKT model to uncover similar trace log terms.

\section{A Orthogonal decomposition of the fluctuations}

To simplify notation let us suppress the time variables. We organize the fluctuations $g_{\mathbf{a}_{\mathbf{c}} \mathbf{b}_{\mathbf{c}}}^{(c)}$ in a column vector with $q$ entries, each of which is a $N^{q-1} \times N^{q-1}$ matrix:

$$
\mathbf{g}=\left(\begin{array}{c}
g_{\mathbf{a}_{\mathbf{1}} \mathbf{b}_{\mathbf{1}}}^{(1)} \\
g_{\mathbf{a}_{\mathbf{2}} \mathbf{b}_{\mathbf{2}}}^{(2)} \\
\vdots \\
g_{\mathbf{a}_{\mathbf{q}} \mathbf{b}_{\mathbf{q}}}^{(q)}
\end{array}\right), \quad\langle\mathbf{h} \mid \mathbf{g}\rangle=\sum_{c=1}^{q} \operatorname{Tr}\left[\left(h^{(c)}\right)^{T} g^{(c)}\right]
$$


where $T$ denotes transposition. We denote $\delta_{\mathbf{a}_{\mathbf{c}} \mathbf{b}_{\mathbf{c}}} \equiv \prod_{c^{\prime} \neq c} \delta_{a_{c c^{\prime}} b_{c c^{\prime}}}$ and $\delta_{\mathbf{a}_{\mathbf{c}} \mathbf{b}_{\mathbf{c}}}^{c-c c_{1}} \equiv$ $\prod_{c^{\prime} \neq c, c_{1}} \delta_{a_{c c^{\prime}} b_{c c^{\prime}}}$. The identity operator in this vector space writes:

$$
\mathbb{I}=\left(\begin{array}{ccc}
I^{(1)} & 0 & 0 \\
0 & \ddots & 0 \\
0 & 0 & I^{(q)}
\end{array}\right), \quad I_{\mathbf{a}_{\mathbf{c}} \mathbf{b}_{\mathbf{c}} ; \mathbf{m}_{\mathbf{c}} \mathbf{n}_{\mathbf{c}}}^{(c)}=\delta_{\mathbf{a}_{\mathbf{c}} \mathbf{m}_{\mathbf{c}}} \delta_{\mathbf{b}_{\mathbf{c}} \mathbf{n}_{\mathbf{c}}}
$$

The 4-point kernel is the operator:

$$
\begin{aligned}
& \lambda^{2} \mathbb{K}=\lambda^{2}\left(\begin{array}{cccc}
0 & K^{(12)} & \ldots & K^{(1 q)} \\
K^{(21)} & 0 & \ldots & K^{(2 q)} \\
\vdots & & & \\
K^{(q 1)} & K^{(q 2)} & \ldots & 0
\end{array}\right)
\end{aligned}
$$

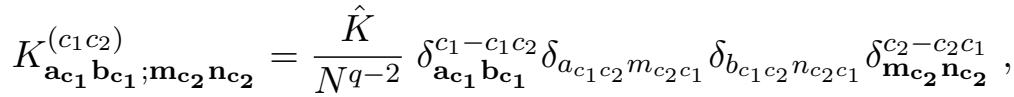

and the Hessian of (4.52) is proportional to the linear operator $\mathbb{I}-\lambda^{2} \mathbb{K}$ on this vector space. Notice that when $K^{\left(c_{1} c_{2}\right)}$ acts on $g_{\mathbf{m}_{\mathbf{c}_{2}} \mathbf{n}_{\mathbf{c}_{\mathbf{2}}}}^{\left(c_{2}\right)}$ it traces it on $q-2$ indices, i.e. those of color different from $c_{1} c_{2}$ (see again figure 11), thus showing the way to its partial diagonalization. We introduce the following operators:

$$
\begin{aligned}
& P_{\mathbf{a}_{\mathbf{c}_{1}} \mathbf{b}_{\mathbf{c}_{\mathbf{1}}} ; \mathbf{m}_{\mathbf{c}_{\mathbf{2}}} \mathbf{n}_{\mathbf{c}_{\mathbf{2}}}}^{\left(c_{1} c_{2}\right.}=\frac{1}{N^{q-1}} \delta_{\mathbf{a}_{\mathbf{c}_{\mathbf{1}}} \mathbf{b}_{\mathbf{c}_{\mathbf{1}}}} \delta_{\mathbf{m}_{\mathbf{c}_{\mathbf{2}}} \mathbf{n}_{\mathbf{c}_{\mathbf{2}}}}, \\
& T_{\mathbf{a}_{\mathbf{c}_{\mathbf{1}}} \mathbf{b}_{\mathbf{c}_{\mathbf{1}}} ; \mathbf{m}_{\mathbf{c}_{\mathbf{2}}} \mathbf{n}_{\mathbf{c}_{\mathbf{2}}}}^{\left(c_{2} c_{2}\right.}=\frac{1}{N^{q-2}} \delta_{\mathbf{a}_{\mathbf{c}_{\mathbf{1}}} \mathbf{b}_{\mathbf{c}_{\mathbf{1}}}}^{c_{1}-c_{1} c_{2}}\left(\delta_{a_{c_{1} c_{2}} m_{c_{2} c_{1}}} \delta_{b_{c_{1} c_{2}} n_{c_{2} c_{1}}}-\frac{1}{N} \delta_{a_{c_{1} c_{2}} b_{c_{1} c_{2}}} \delta_{m_{c_{2} c_{1}} n_{c_{2} c_{1}}}\right) \delta_{\mathbf{m}_{\mathbf{c}_{\mathbf{2}}} \mathbf{n}_{\mathbf{c}_{\mathbf{2}}}}^{c_{2}-c_{2} c_{1}},
\end{aligned}
$$

and:

$$
R_{\mathbf{a}_{\mathbf{c}} \mathbf{b}_{\mathbf{c}} ; \mathbf{m}_{\mathbf{c}} \mathbf{n}_{\mathbf{c}}}^{(c-c c}=\frac{1}{N^{q-2}} \delta_{\mathbf{a}_{\mathbf{c}} \mathbf{b}_{\mathbf{c}}}^{c-c c_{1}}\left(\delta_{a_{c c_{1}} m_{c c_{1}}} \delta_{b_{c c_{1}} n_{c c_{1}}}-\frac{1}{N} \delta_{a_{c c_{1}} b_{c c_{1}}} \delta_{m_{c c_{1}} n_{c c_{1}}}\right) \delta_{\mathbf{m}_{\mathbf{c}} \mathbf{n}_{\mathbf{c}}}^{c-c c_{1}}
$$

In words, when acting on a fluctuations $g^{\left(c_{2}\right)}, P^{\left(c_{1} c_{2}\right)}$ traces all the indices and replaces them with an identity on color $c_{1} ; T^{\left(c_{1} c_{2}\right)}$ does the same but spares the shared color $\left(c_{1} c_{2}\right)$, on which it projects on the traceless part; lastly, $R^{\left(c_{2}-c_{2} c_{1}\right)}$ is similar to $T^{\left(c_{1} c_{2}\right)}$, but it does not change the color of the traced indices. They satisfy (no sum over $c$ ):

$$
\begin{aligned}
P^{\left(c_{1} c\right)} P^{\left(c c_{2}\right)} & =P^{\left(c_{1} c_{2}\right)}, & P^{\left(c_{1} c\right)} T^{\left(c c_{2}\right)} & =T^{\left(c_{1} c\right)} P^{\left(c c_{2}\right)}=0, \\
T^{\left(c_{1} c\right)} T^{\left(c c_{2}\right)} & =R^{\left(c_{2}-c_{2} c\right)} \delta_{c_{1} c_{2}}, & T^{\left(c_{1} c\right)} R^{\left(c-c c_{2}\right)} & =T^{\left(c_{1} c\right)} \delta_{c_{1} c_{2}} \\
R^{\left(c-c c_{1}\right)} R^{\left(c-c c_{2}\right)} & =R^{\left(c-c c_{2}\right)} \delta_{c_{1} c_{2}}, & P^{\left(c_{1} c\right)} R^{\left(c-c c_{2}\right)} & =R^{\left(c-c c_{1}\right)} P^{\left(c c_{2}\right)}=0 .
\end{aligned}
$$

In the vector space spanned by $\mathbf{g}$ the 4-point kernel splits as the sum of two operators $\mathbb{K}=\hat{K}(\mathbb{T}+\mathbb{P})$, with:

$$
\mathbb{P}=\left(\begin{array}{cccc}
0 & P^{(12)} & \ldots & P^{(1 q)} \\
P^{(21)} & 0 & \ldots & P^{(2 q)} \\
\vdots & & & \\
P^{(q 1)} & P^{(q 2)} & \ldots & 0
\end{array}\right), \quad \mathbb{T}=\left(\begin{array}{cccc}
0 & T^{(12)} & \ldots & T^{(1 q)} \\
T^{(21)} & 0 & \ldots & T^{(2 q)} \\
\vdots & & & \\
T^{(q 1)} & T^{(q 2)} & \ldots & 0
\end{array}\right) .
$$


Introducing also the projectors:

$$
\mathbb{Q}=\left(\begin{array}{ccc}
P^{(11)} & 0 & 0 \\
0 & \ddots & 0 \\
0 & 0 & P^{(q q)}
\end{array}\right), \quad \mathbb{R}=\mathbb{T}^{2}=\left(\begin{array}{ccc}
\sum_{c \neq 1} R^{(1-1 c)} & 0 & 0 \\
0 & \ddots & 0 \\
0 & 0 & \sum_{c \neq q} R^{(q-q c)}
\end{array}\right),
$$

the identity can be decomposed in orthogonal components as $\mathbb{I}=\hat{\mathbb{I}}+\mathbb{Q}+\mathbb{R}$, where $\hat{\mathbb{I}}=$ $\mathbb{I}-\mathbb{Q}-\mathbb{R}$. Using such a decomposition of the identity we can write:

$$
\mathbf{g}=\mathbb{Q} \mathbf{g}+\mathbb{R} \mathbf{g}+\hat{\mathbb{I}} \mathbf{g},
$$

which in components is:

$$
g_{\mathbf{a}_{\mathbf{c}} \mathbf{b}_{\mathbf{c}}}^{(c)}=g^{(c)} \delta_{\mathbf{a}_{\mathbf{c}} \mathbf{b}_{\mathbf{c}}}^{c}+\sum_{c_{1} \neq c} g_{a_{c c_{1}} b_{c c_{1}}}^{\left(c c_{1}\right)} \delta_{\mathbf{a}_{\mathbf{c}} \mathbf{b}_{\mathbf{c}}}^{c-c c c_{1}}+\hat{g}_{\mathbf{a}_{\mathbf{c}} \mathbf{b}_{\mathbf{c}}}^{(c)},
$$

with:

$$
\begin{aligned}
g^{(c)} & =\frac{1}{N^{q-1}} \delta_{\mathbf{m}_{\mathbf{c}} \mathbf{n}_{\mathbf{c}}}^{c} g_{\mathbf{m}_{\mathbf{c}} \mathbf{n}_{\mathbf{c}}}^{(c)}, \\
g_{a_{c c_{1}} b_{c c_{1}}}^{\left(c c_{1}\right)} & =\frac{1}{N^{q-2}}\left(\delta_{a_{c c_{1}} m_{c c_{1}}} \delta_{b_{c c_{1}} n_{c c_{1}}}-\frac{1}{N} \delta_{a_{c c_{1}} b_{c c_{1}}} \delta_{m_{c c_{1}} n_{c c_{1}}}\right) \delta_{\mathbf{m}_{\mathbf{c}} \mathbf{n}_{\mathbf{c}}}^{c-c c_{1}} g_{\mathbf{m}_{\mathbf{c}} \mathbf{n}_{\mathbf{c}}}^{(c)}, \\
\delta_{\mathbf{a}_{\mathbf{c}} \mathbf{b}_{\mathbf{c}}}^{c-c c_{1}} \hat{g}_{\mathbf{a}_{\mathbf{c}} \mathbf{b}_{\mathbf{c}}}^{(c)} & =0
\end{aligned}
$$

which is the decomposition introduced in (4.55).

The quadratic action for the fluctuations thus writes:

$$
\left\langle\mathbf{g}\left|\left(\mathbb{I}-\lambda^{2} \mathbb{K}\right)\right| \mathbf{g}\right\rangle=\left\langle\mathbb{Q} \mathbf{g}\left|\left(\mathbb{Q}-\lambda^{2} \hat{K} \mathbb{P}\right)\right| \mathbb{Q} \mathbf{g}\right\rangle+\left\langle\mathbb{R} \mathbf{g}\left|\left(\mathbb{R}-\lambda^{2} \hat{K} \mathbb{T}\right)\right| \mathbb{R} \mathbf{g}\right\rangle+\langle\hat{\mathbb{I}} \mathbf{g} \mid \hat{\mathbb{I}} \mathbf{g}\rangle .
$$

Furthermore, we can decompose:

$$
\left\langle\mathbb{R} \mathbf{g}\left|\left(\mathbb{R}-\lambda^{2} \hat{K} \mathbb{T}\right)\right| \mathbb{R} \mathbf{g}\right\rangle=N^{q-2} \sum_{c_{1}<c_{2}}\left(g^{\left(c_{1} c_{2}\right)} g^{\left(c_{2} c_{1}\right)}\right)\left(\begin{array}{cc}
1 & -\lambda^{2} \hat{K} \\
-\lambda^{2} \hat{K} & 1
\end{array}\right)\left(\begin{array}{l}
g^{\left(c_{1} c_{2}\right)} \\
g^{\left(c_{2} c_{1}\right)}
\end{array}\right) .
$$

Lastly, reintroducing the time variables, we notice that given that $g_{a b}^{\left(c c^{\prime}\right)}\left(t, t^{\prime}\right)=-g_{b a}^{\left(c c^{\prime}\right)}\left(t^{\prime}, t\right)$ , we can rewrite (omitting the subscript $c c^{\prime}$ on the indices):

$$
g_{a b}^{\left(c c^{\prime}\right)}\left(t, t^{\prime}\right) g_{a b}^{\left(c c^{\prime}\right)}\left(t, t^{\prime}\right)=\int_{s, s^{\prime}} g_{a b}^{\left(c c^{\prime}\right)}\left(t, t^{\prime}\right) \Im_{a b ; m n}\left(t, t^{\prime} ; s, s^{\prime}\right) g_{m n}^{\left(c c^{\prime}\right)}\left(s, s^{\prime}\right)
$$

where:

$$
\Im_{a b ; m n}\left(t, t^{\prime} ; s, s^{\prime}\right)=\mathcal{S}_{a b ; m n} I_{-}\left(t, t^{\prime} ; s, s^{\prime}\right)+\mathcal{A}_{a b ; m n} I_{+}\left(t, t^{\prime} ; s, s^{\prime}\right)
$$

and:

$$
\begin{aligned}
\mathcal{S}_{a b ; m n} & =\frac{1}{2}\left(\delta_{a m} \delta_{b n}+\delta_{a n} \delta_{b m}\right), \\
\mathcal{A}_{a b ; m n} & =\frac{1}{2}\left(\delta_{a m} \delta_{b n}-\delta_{a n} \delta_{b m}\right), \\
I_{ \pm}\left(t, t^{\prime} ; s, s^{\prime}\right) & =\frac{1}{2}\left(\delta(t-s) \delta\left(t^{\prime}-s^{\prime}\right) \pm \delta\left(t-s^{\prime}\right) \delta\left(t^{\prime}-s\right)\right) .
\end{aligned}
$$

Together, (A.12), (A.13), and (A.14) realize the block-diagonalization described in the text, thus leading to the trace $\log$ terms in (4.51) upon integration over the fluctuations. ${ }^{17}$

\footnotetext{
${ }^{17}$ Plus some constant factors (in particular logarithmic terms in $N$ coming for example from the $N^{q-2}$ factor in (A.13)), which can be absorbed in the measure.
} 
Open Access. This article is distributed under the terms of the Creative Commons Attribution License (CC-BY 4.0), which permits any use, distribution and reproduction in any medium, provided the original author(s) and source are credited.

\section{References}

[1] E. Witten, An SYK-like model without disorder, arXiv:1610.09758 [INSPIRE].

[2] S. Sachdev and J. Ye, Gapless spin fluid ground state in a random, quantum Heisenberg magnet, Phys. Rev. Lett. 70 (1993) 3339 [cond-mat/9212030] [INSPIRE].

[3] A. Kitaev, A simple model of quantum holography, talks given at KITP, April 7 and May 27, KITP, U.S.A. (2015).

[4] J. Polchinski and V. Rosenhaus, The spectrum in the Sachdev-Ye-Kitaev model, JHEP 04 (2016) 001 [arXiv: 1601.06768] [INSPIRE].

[5] J. Maldacena and D. Stanford, Remarks on the Sachdev-Ye-Kitaev model, Phys. Rev. D 94 (2016) 106002 [arXiv: 1604.07818] [INSPIRE].

[6] D.J. Gross and V. Rosenhaus, A generalization of Sachdev-Ye-Kitaev, JHEP 02 (2017) 093 [arXiv: 1610.01569] [INSPIRE].

[7] A. Kitaev and S.J. Suh, The soft mode in the Sachdev-Ye-Kitaev model and its gravity dual, arXiv: 1711.08467 [INSPIRE].

[8] S. Sachdev, Bekenstein-Hawking entropy and strange metals, Phys. Rev. X 5 (2015) 041025 [arXiv: 1506.05111] [INSPIRE].

[9] A. Jevicki, K. Suzuki and J. Yoon, Bi-local holography in the SYK model, JHEP 07 (2016) 007 [arXiv: 1603.06246] [INSPIRE].

[10] K. Jensen, Chaos in AdS 2 holography, Phys. Rev. Lett. 117 (2016) 111601 [arXiv: 1605.06098] [INSPIRE].

[11] J. Maldacena, D. Stanford and Z. Yang, Conformal symmetry and its breaking in two dimensional nearly Anti-de-Sitter space, PTEP 2016 (2016) 12C104 [arXiv:1606.01857] [INSPIRE].

[12] J. Engelsöy, T.G. Mertens and H. Verlinde, An investigation of $A d S_{2}$ backreaction and holography, JHEP 07 (2016) 139 [arXiv:1606.03438] [INSPIRE].

[13] A. Jevicki and K. Suzuki, Bi-local holography in the SYK model: perturbations, JHEP 11 (2016) 046 [arXiv: 1608.07567] [INSPIRE].

[14] D.J. Gross and V. Rosenhaus, The bulk dual of SYK: cubic couplings, JHEP 05 (2017) 092 [arXiv: 1702.08016] [INSPIRE].

[15] D.J. Gross and V. Rosenhaus, All point correlation functions in SYK, JHEP 12 (2017) 148 [arXiv: 1710.08113] [INSPIRE].

[16] S.R. Das and A. Jevicki, Large $N$ collective fields and holography, Phys. Rev. D 68 (2003) 044011 [hep-th/0304093] [INSPIRE].

[17] R. de Mello Koch, A. Jevicki, K. Jin and J.P. Rodrigues, $A d S_{4} / C F T_{3}$ construction from collective fields, Phys. Rev. D 83 (2011) 025006 [arXiv:1008.0633] [INSPIRE].

[18] R. Gurau, Quenched equals annealed at leading order in the colored SYK model, EPL 119 (2017) 30003 [arXiv: 1702.04228] [INSPIRE]. 
[19] R. Gurau, Colored group field theory, Commun. Math. Phys. 304 (2011) 69 [arXiv: 0907.2582] [INSPIRE].

[20] R. Gurau, Random tensors, Oxford University Press, Oxford U.K. (2016).

[21] C. Peng, M. Spradlin and A. Volovich, A supersymmetric SYK-like tensor model, JHEP 05 (2017) 062 [arXiv: 1612.03851] [INSPIRE].

[22] I.R. Klebanov and G. Tarnopolsky, Uncolored random tensors, melon diagrams and the Sachdev-Ye-Kitaev models, Phys. Rev. D 95 (2017) 046004 [arXiv:1611.08915] [InSPIRE].

[23] I.R. Klebanov and G. Tarnopolsky, On large $N$ limit of symmetric traceless tensor models, JHEP 10 (2017) 037 [arXiv: 1706.00839] [INSPIRE].

[24] S. Giombi, I.R. Klebanov and G. Tarnopolsky, Bosonic tensor models at large $N$ and small $\epsilon$, Phys. Rev. D 96 (2017) 106014 [arXiv:1707.03866] [InSPIRE].

[25] K. Bulycheva, I.R. Klebanov, A. Milekhin and G. Tarnopolsky, Spectra of operators in large $N$ tensor models, Phys. Rev. D 97 (2018) 026016 [arXiv:1707.09347] [INSPIRE].

[26] M. Beccaria and A.A. Tseytlin, Partition function of free conformal fields in 3-plet representation, JHEP 05 (2017) 053 [arXiv: 1703.04460] [INSPIRE].

[27] S. Choudhury et al., Notes on melonic $O(N)^{q-1}$ tensor models, arXiv:1707.09352 [INSPIRE].

[28] S. Prakash and R. Sinha, A complex fermionic tensor model in d dimensions, JHEP 02 (2018) 086 [arXiv : 1710.09357] [INSPIRE].

[29] D. Benedetti, S. Carrozza, R. Gurau and A. Sfondrini, Tensorial Gross-Neveu models, JHEP 01 (2018) 003 [arXiv:1710.10253] [INSPIRE].

[30] C. Peng, Vector models and generalized SYK models, JHEP 05 (2017) 129 [arXiv: 1704.04223] [INSPIRE].

[31] N. Halmagyi and S. Mondal, Tensor models for black hole probes, arXiv:1711.04385 [INSPIRE].

[32] C. Krishnan, S. Sanyal and P.N. Bala Subramanian, Quantum chaos and holographic tensor models, JHEP 03 (2017) 056 [arXiv:1612.06330] [INSPIRE].

[33] C. Krishnan and K.V.P. Kumar, Towards a finite-N hologram, JHEP 10 (2017) 099 [arXiv: 1706.05364] [INSPIRE].

[34] C. Krishnan, K.V. Pavan Kumar and D. Rosa, Contrasting SYK-like models, JHEP 01 (2018) 064 [arXiv: 1709.06498] [inSPIRE].

[35] C. Krishnan and K.V. Pavan Kumar, Exact solution of a strongly coupled gauge theory in $0+1$ dimensions, Phys. Rev. Lett. 120 (2018) 201603 [arXiv: 1802.02502] [INSPIRE].

[36] F. Ferrari, The large D limit of planar diagrams, arXiv:1701.01171 [INSPIRE].

[37] T. Azeyanagi, F. Ferrari and F.I. Schaposnik Massolo, Phase diagram of planar matrix quantum mechanics, tensor and Sachdev-Ye-Kitaev models, Phys. Rev. Lett. 120 (2018) 061602 [arXiv: 1707.03431] [INSPIRE].

[38] F. Ferrari, V. Rivasseau and G. Valette, A new large $N$ expansion for general matrix-tensor models, arXiv: 1709.07366 [INSPIRE].

[39] T. Azeyanagi, F. Ferrari, P. Gregori, L. Leduc and G. Valette, More on the new large D limit of matrix models, Annals Phys. 393 (2018) 308 [arXiv:1710.07263] [INSPIRE]. 
[40] V. Bonzom, L. Lionni and A. Tanasa, Diagrammatics of a colored SYK model and of an SYK-like tensor model, leading and next-to-leading orders, J. Math. Phys. 58 (2017) 052301 [arXiv: 1702.06944] [INSPIRE].

[41] V. Rivasseau, Constructive tensor field theory, SIGMA 12 (2016) 085 [arXiv:1603.07312] [INSPIRE].

[42] J. Ben Geloun and V. Rivasseau, A renormalizable SYK-type tensor field theory, arXiv: 1711.05967 [INSPIRE].

[43] J. Ben Geloun, R. Martini and D. Oriti, Functional renormalisation group analysis of tensorial group field theories on $\mathbb{R}^{d}$, Phys. Rev. D 94 (2016) 024017 [arXiv:1601.08211] [INSPIRE].

[44] J. Ben Geloun and S. Ramgoolam, Counting tensor model observables and branched covers of the 2-sphere, arXiv:1307.6490 [INSPIRE].

[45] J. Ben Geloun and S. Ramgoolam, Tensor models, Kronecker coefficients and permutation centralizer algebras, JHEP 11 (2017) 092 [arXiv: 1708.03524] [INSPIRE].

[46] R. de Mello Koch, R. Mello Koch, D. Gossman and L. Tribelhorn, Gauge invariants, correlators and holography in bosonic and fermionic tensor models, JHEP 09 (2017) 011 [arXiv: 1707.01455] [INSPIRE].

[47] E. Gardner, Spin glasses with p-spin interactions, Nucl. Phys. B 257 (1985) 747 [INSPIRE].

[48] L.F. Cugliandolo, D.R. Grempel and C.A. da Silva Santos, Imaginary-time replica formalism study of a quantum spherical p-spin-glass model, articlePhys. Rev. B 64 (2001) 014403 [cond-mat/0012222].

[49] A. Jevicki and B. Sakita, Collective field approach to the large $N$ limit: euclidean field theories, Nucl. Phys. B 185 (1981) 89 [InSPIRE].

[50] S. Dartois, H. Erbin and S. Mondal, Conformality of $1 / N$ corrections in SYK-like models, arXiv:1706.00412 [INSPIRE].

[51] J.M. Cornwall, R. Jackiw and E. Tomboulis, Effective action for composite operators, Phys. Rev. D 10 (1974) 2428 [INSPIRE].

[52] J. Berges, Introduction to nonequilibrium quantum field theory, AIP Conf. Proc. 739 (2005) 3 [hep-ph/0409233] [INSPIRE].

[53] J.P. Blaizot, E. Iancu and A. Rebhan, Approximately selfconsistent resummations for the thermodynamics of the quark gluon plasma. 1. Entropy and density, Phys. Rev. D 63 (2001) 065003 [hep-ph/0005003] [INSPIRE].

[54] J. Berges, S. Borsányi, U. Reinosa and J. Serreau, Renormalized thermodynamics from the 2PI effective action, Phys. Rev. D 71 (2005) 105004 [hep-ph/0409123] [INSPIRE].

[55] J. Berges, S. Borsányi, U. Reinosa and J. Serreau, Nonperturbative renormalization for 2PI effective action techniques, Annals Phys. 320 (2005) 344 [hep-ph/0503240] [INSPIRE].

[56] U. Reinosa and J. Serreau, 2PI functional techniques for gauge theories: QED, Annals Phys. 325 (2010) 969 [arXiv:0906.2881] [INSPIRE].

[57] J. Berges, Controlled nonperturbative dynamics of quantum fields out-of-equilibrium, Nucl. Phys. A 699 (2002) 847 [hep-ph/0105311] [INSPIRE]. 
[58] G. Aarts, D. Ahrensmeier, R. Baier, J. Berges and J. Serreau, Far from equilibrium dynamics with broken symmetries from the $2 P I-1 / N$ expansion, Phys. Rev. D 66 (2002) 045008 [hep-ph/0201308] [INSPIRE].

[59] S. Carrozza and A. Tanasa, $O(N)$ random tensor models, Lett. Math. Phys. 106 (2016) 1531 [arXiv: 1512.06718] [INSPIRE].

[60] N.D. Mermin and H. Wagner, Absence of ferromagnetism or antiferromagnetism in one-dimensional or two-dimensional isotropic Heisenberg models, Phys. Rev. Lett. 17 (1966) 1133 [INSPIRE].

[61] S.R. Coleman, There are no Goldstone bosons in two-dimensions, Commun. Math. Phys. 31 (1973) 259 [INSPIRE].

[62] E. Witten, Chiral symmetry, the $1 / n$ expansion and the $\mathrm{SU}(N)$ Thirring model, Nucl. Phys. B 145 (1978) 110 [INSPIRE].

[63] J. Magnen and V. Rivasseau, Constructive $\phi^{4}$ field theory without tears, Annales Henri Poincaré 9 (2008) 403 [arXiv:0706.2457] [INSPIRE].

[64] P. Di Vecchia, M. Kato and N. Ohta, Double scaling limit in $O(N)$ vector models, Nucl. Phys. B 357 (1991) 495 [INSPIRE].

[65] D. Benedetti, S. Carrozza, R. Gurau and M. Kolanowski, The $1 / N$ expansion of the symmetric traceless and the antisymmetric tensor models in rank three, arXiv:1712.00249 [INSPIRE].

[66] R. Gurau and G. Schaeffer, Regular colored graphs of positive degree, Ann. Inst. Henri Poincaré Comb. Phys. Interact. 3 (2016) 257 [arXiv:1307.5279]. 